\title{
Collapse Dynamics in Hilbert-space Stochastic Processes
}

\section{Daniele Bajoni}

University of Pavia

Oreste Nicrosini

INFN Sezione di Pavia

Alberto Rimini

University of Pavia

Simone Rodini ( $\nabla$ simone.rodini01@universitadipavia.it )

University of Pavia

\section{Research Article}

Keywords: Hilbert-space stochastic processes, Ghirardi-Rimini-Weber, spontaneous collapse, continuous realisations

Posted Date: May 12th, 2021

DOl: https://doi.org/10.21203/rs.3.rs-512683/v1

License: (1) (1) This work is licensed under a Creative Commons Attribution 4.0 International License. Read Full License

Version of Record: A version of this preprint was published at Scientific Reports on November 12th, 2021. See the published version at https://doi.org/10.1038/s41598-021-00737-1. 


\title{
Collapse dynamics in Hilbert-space stochastic processes
}

\author{
Daniele Bajoni, ${ }^{1}$ Oreste Nicrosini, ${ }^{2,3}$ Alberto Rimini, ${ }^{2,3}$ and Simone Rodini*2,3 \\ ${ }^{1}$ Dipartimento di Ingegneria Industriale e dell'Informazione - Università di Pavia - Italy \\ ${ }^{2}$ Istituto Nazionale di Fisica Nucleare - Sezione di Pavia - Italy \\ ${ }^{3}$ Dipartimento di Fisica - Università di Pavia - Italy
}

(Dated: May 10, 2021)

\begin{abstract}
A detailed study of collapse dynamics as foreseen by the Ghirardi-Rimini-Weber theory of spontaneous collapse and its continuous realisations in the form of stochastic differential equations describing a brownian motion in the Hilbert space is presented.
\end{abstract}




\section{INTRODUCTION}

In attempting to propose possible solutions to the quantum measurement problem, a number of possible solutions have been proposed. On the one hand, there exist many decoherence theories, see ref. [14] for a review. On the other hand, different extensions of quantum mechanics have been developed. On the one side, discontinuous stochastic processes promoting the spontaneous collapse of the state of the quantum system have been presented for the first time in ref. [8], namely the so called GRW theory. On the other one, continuous processes such as in ref. [7] have been devised, in which the spontaneous collapse of the quantum state is realised in the form of a brownian motion in the Hilbert space. In both the approaches, the spontaneous collapse originates from stochastic and nonlinear terms additional with respect to the deterministic and linear Schrödinger evolution, see refs. $[3,5-9,16])$.

The two families of processes are strictly connected. Actually, in ref. [12] it has been shown that continuous processes can be obtained as a proper infinite frequency limit of discontinuous ones. In all these process in the Hilbert space a set of physical quantities (observables) appears, represented by the corresponding set of selfadjoint operators. The processes act inducing the sharpening of the distribution of values of those quantities around a stochastically chosen centre. In ref. [6] it has been shown that the physical effect of the stochastic processes depends on the choice of observables that are being sharpened and not on the details of the sharpening procedure.

The two approaches, being formally distinct, have different features. On the one hand, the meaning of the parameters and the physical effects of the sharpening procedure are clearer in the discontinuous processes; on the other one, the continuous realisations are more elegant from the mathematical viewpoint. The paper is organised as follows. In Sect. II the main features of the two approaches are recalled, together with their relationship. In Sect. III the continuous process is specialised to an experimental setup in which a single photon is sent to a beam splitter creating a superposition of transmitted/reflected photon states, and the photon is detected (or not) by a single-photon detector placed in the transmission region. Sect. IV is devoted to the analysis of the setup in which a second single-photon detector is added in the reflection region. In Sect. $\mathrm{V}$ the photon/detector interaction is modelled and taken into account in the measurement dynamics, together with its interplay with the 
stochastic reduction process. In Sect. VI a number numerical simulations are shown and commented. In Sect. VII some conclusions are drawn.

\section{HITTING AND CONTINUOUS PROCESSES}

Let the set of compatible quantities characterizing the discontinuous stochastic process be

$$
\hat{\boldsymbol{A}} \equiv\left\{\hat{A}_{m} ; m=1,2, \ldots, K\right\}, \quad\left[\hat{A}_{m}, \hat{A}_{n}\right]=0, \quad \hat{A}_{m}^{\dagger}=\hat{A}_{m},
$$

and the sharpening action be given by the operator

$$
S_{i}=\left(\frac{\alpha}{\pi}\right)^{K / 4} \exp \left(R_{i}\right), \quad R_{i}=-\frac{1}{2} \alpha\left(\hat{\boldsymbol{A}}-\boldsymbol{a}_{i}\right)^{2} .
$$

The parameter $\alpha$ rules the accuracy of the sharpening and $\boldsymbol{a}_{i}$ is the centre of the $i$-th hitting. It is assumed that the hittings occur randomly in time, distributed according to a Poisson law with frequency $\lambda$.

The sharpening operator for the $i$-th hitting $S_{i}$ acts on the normalized state vector $\left|\psi_{t}\right\rangle$ giving the state vector $\left|\varphi_{i, t}\right\rangle$, which can be recast in a normalized vector $\left|\psi_{i, t}^{\prime}\right\rangle$ :

$$
\left|\psi_{i, t}^{\prime}\right\rangle=\frac{\left|\varphi_{i, t}\right\rangle}{\left\|\varphi_{i, t}\right\|}, \quad\left|\varphi_{i, t}\right\rangle=S_{i}\left|\psi_{t}\right\rangle
$$

The probability that the hitting takes place around $\boldsymbol{a}_{i}$ is

$$
\mathscr{P}\left(\psi_{t} \mid \boldsymbol{a}_{i}\right)=\left\|\varphi_{i, t}\right\|^{2}
$$

Actually, it turns out that the effectiveness of the discontinuous process depends on $\alpha$ and $\lambda$ only through their product.

The continuous process based on the same quantities $\hat{\boldsymbol{A}} \equiv\left\{\hat{A}_{m}\right\}_{m=1}^{K}$ is ruled by the Itô stochastic differential equation

$$
\mathrm{d}|\psi\rangle=\left[\sqrt{\gamma}\left(\hat{\boldsymbol{A}}-\langle\hat{\boldsymbol{A}}\rangle_{\psi_{t}}\right) \cdot \mathrm{d} \boldsymbol{B}-\frac{1}{2} \gamma\left(\hat{\boldsymbol{A}}-\langle\hat{\boldsymbol{A}}\rangle_{\psi_{t}}\right)^{2} \mathrm{~d} t\right]|\psi\rangle,
$$

where

$$
\mathrm{d} \boldsymbol{B} \equiv\left\{\mathrm{d} B_{m} ; m=1,2, \ldots, K\right\}, \quad \overline{\mathrm{d} \boldsymbol{B}}=0, \quad \overline{\mathrm{d} B_{m} \mathrm{~d} B_{n}}=\delta_{m n} \mathrm{~d} t
$$

and

$$
\langle\hat{\boldsymbol{A}}\rangle_{\psi_{t}}=\left\langle\psi_{t}|\hat{\boldsymbol{A}}| \psi_{t}\right\rangle
$$


The parameter $\gamma$ sets the effectiveness of the process.

In refs. [12] and [6] it has been shown that, by taking the infinite frequency limit of the discontinuous process (3) and (4) with the prescription

$$
\alpha \lambda=\text { constant }=2 \gamma
$$

one gets the continuous process of eq. (5). As a consequence it becomes apparent that, for $t \rightarrow \infty$, the continuous process drives the state vector to a common eigenvector of the operators $\hat{\boldsymbol{A}}$. The probability of a particular eigenvector $\left|\boldsymbol{a}_{r}\right\rangle$ is given by $\left|\left\langle\boldsymbol{a}_{r} \mid \psi_{0}\right\rangle\right|^{2}$, for the generic state vector $\left|\psi_{0}\right\rangle$ at a given arbitrary initial time.

\section{SINGLE DETECTOR}

Consider the experimental setup depicted in Figure 1, in which a single photon state hits a beam splitter (BS), a superposition of $|\gamma\rangle_{R}$ and $|\gamma\rangle_{L}$ states is formed and a single photon detector (SPD) is placed in $R$ position. In this case eqs. (5)-(7) are specialized to a single operator $\hat{A}$, namely the operator associated to the "pointer position" (more on this later).

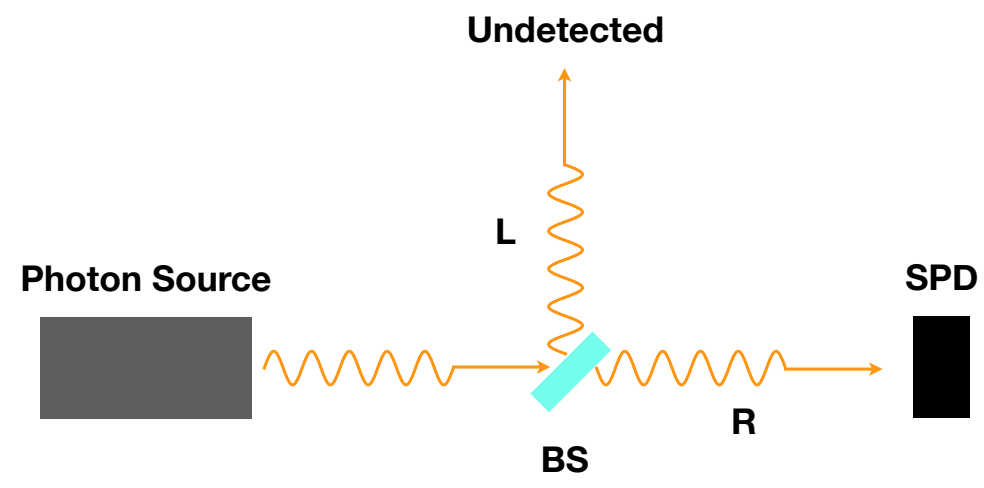

FIG. 1: Single detector setup.

We indicate with $\left|c_{R, L}\right|^{2}$ the transmission and reflection coefficients of the BS, respectively. Therefore, in the photon + detector Hilbert space one has that the Schrödinger evolution 
generates the superposition

$$
\begin{aligned}
|\psi\rangle=\left(c_{L}|\gamma\rangle_{L}+c_{R}|\gamma\rangle_{R}\right)\left|D^{(0)}\right\rangle & \rightarrow c_{L}|\gamma\rangle_{L}\left|D^{(0)}\right\rangle+c_{R}|\gamma\rangle_{R}\left|D^{(+)}\right\rangle \\
& =c_{L}\left|\psi_{L}\right\rangle+c_{R}\left|\psi_{R}\right\rangle
\end{aligned}
$$

where $\left|D^{(0,+)}\right\rangle$ are the SPD states that correspond to SPD "ready" or "clicked", respectively, and $\left|c_{L}\right|^{2}+\left|c_{R}\right|^{2}=1$. The operator associated to the pointer position can be represented as

$$
\hat{A}=\mathbb{1}_{\gamma} \otimes\left[a_{0}\left|D^{(0)}\right\rangle\left\langle D^{(0)}\left|+a_{+}\right| D^{(+)}\right\rangle\left\langle D^{(+)}\right|\right]
$$

where $a_{0,+}$ are the pointer position eigenvalues. Eqs. (5)-(7) become

$$
\begin{aligned}
\mathrm{d}|\psi\rangle & =\left[\sqrt{\gamma}\left(\hat{A}-\langle\hat{A}\rangle_{\psi_{t}}\right) \mathrm{d} B-\frac{1}{2} \gamma\left(\hat{A}-\langle\hat{A}\rangle_{\psi_{t}}\right)^{2} \mathrm{~d} t\right]|\psi\rangle, \\
\overline{\mathrm{d} B} & =0, \quad \overline{(\mathrm{d} B)^{2}}=\mathrm{d} t, \\
\langle\hat{A}\rangle_{\psi_{t}} & =a_{0}\left|c_{L}\right|^{2}+a_{+}\left|c_{R}\right|^{2}=J(t) .
\end{aligned}
$$

The time evolution of the coefficients of the superposition, $c_{R, L}$, as due to the stochastic process, can be obtained by projecting $|\psi(t+\mathrm{d} t)\rangle$ onto $\left|\psi_{R, L}\right\rangle$, namely

$$
c_{R, L}(t+\mathrm{d} t)=\left\langle\psi_{R, L} \mid \psi(t+\mathrm{d} t)\right\rangle=\left\langle\psi _ { R , L } \left|(|\psi(t)\rangle+\mathrm{d}|\psi\rangle)=L_{R, L}(t) c_{R, L}(t)\right.\right.
$$

where

$$
L_{R, L}(t)=1+\left[\sqrt{\gamma} K_{+, 0}(t) \mathrm{d} B-\frac{1}{2} \gamma K_{+, 0}^{2}(t) \mathrm{d} t\right]
$$

and $K_{0,+}(t)=a_{0,+}-J(t)$. Since $K_{i}(t)$ (and hence $\left.L_{i}(t)\right)$ is invariant under translation of the system of eigenvalues $a_{i}$, without loss of generality one can take $a_{0}=0$ and $a_{+}=a$, obtaining

$$
\begin{aligned}
& K_{0}(t)=-a\left|c_{R}\right|^{2}, \\
& K_{+}(t)=a\left(1-\left|c_{R}\right|^{2}\right)=a\left|c_{L}\right|^{2},
\end{aligned}
$$

from which

$$
L_{R, L}(t)=1+\left[ \pm a \sqrt{\gamma}\left|c_{L, R}(t)\right|^{2} \mathrm{~d} B-\frac{1}{2} \gamma a^{2}\left|c_{L, R}(t)\right|^{4} \mathrm{~d} t\right] .
$$

Since, according to eq. (14),

$$
\left|c_{R, L}(t+\mathrm{d} t)\right|^{2}=L_{R, L}^{2}(t)\left|c_{R, L}(t)\right|^{2}
$$


one has, by squaring eq. (18) and taking into account the Itô lemma, that:

$$
L_{R, L}^{2}(t)=1 \pm 2 a \sqrt{\gamma}\left|c_{L, R}(t)\right|^{2} \mathrm{~d} B
$$

The stochastic factor $L_{R, L}^{2}(t)$ of eq. (20) can be generated numerically in each step in $d t$ by extracting a gaussian random number $\mathrm{d} B$, according to the statistics of eq. (12), and iteratively inserting it into eq. (19) to produce the "path" followed by $\left|c_{R, L}\right|^{2}$ during the reduction process.

\section{TWO DETECTORS}

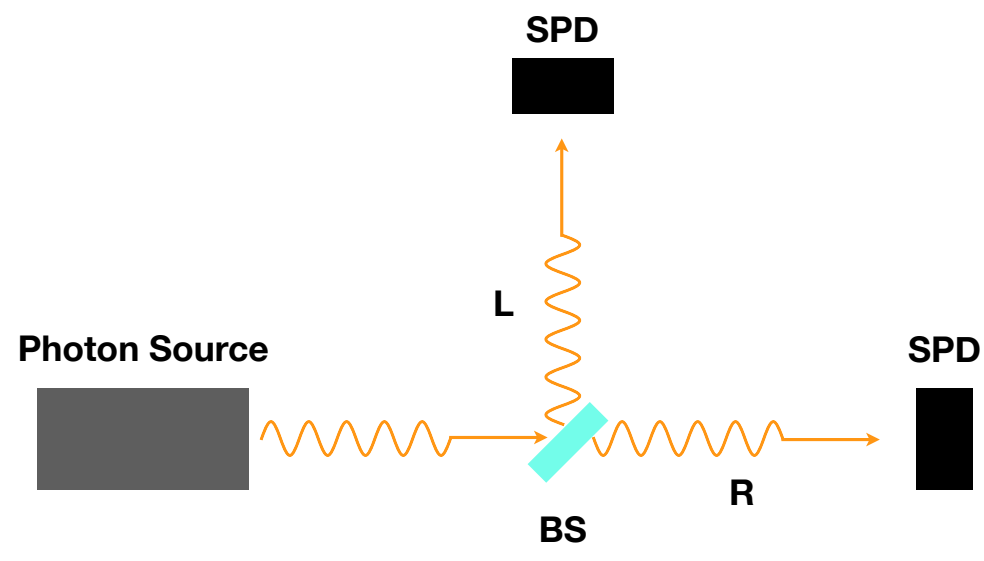

FIG. 2: Double detector setup.

When considering the experimental setup described in Figure 2, where a second (and, for the sake of simplicity, identical) SPD has been added in $L$ position, the Schrödinger evolution generates, in the photon + detectors Hilbert space, the superposition

$$
\begin{aligned}
|\psi\rangle & =\left(c_{L}|\gamma\rangle_{L}+c_{R}|\gamma\rangle_{R}\right)\left|D_{L}^{(0)}\right\rangle\left|D_{R}^{(0)}\right\rangle \\
& \rightarrow c_{L}|\gamma\rangle_{L}\left|D_{L}^{(+)}\right\rangle\left|D_{R}^{(0)}\right\rangle+c_{R}|\gamma\rangle_{R}\left|D_{L}^{(0)}\right\rangle\left|D_{R}^{(+)}\right\rangle=c_{L}\left|\psi_{L}\right\rangle+c_{R}\left|\psi_{R}\right\rangle
\end{aligned}
$$

where now $D_{R, L}$ are the states of the $R$ and $L \mathrm{SPD}$, respectively, and, again, $\left|c_{L}\right|^{2}+\left|c_{R}\right|^{2}=1$. 
The operators associated to the $R$ and $L$ pointer position are

$$
\hat{A}_{R, L}=\mathbb{1}_{\gamma} \otimes \mathbb{1}_{L, R} \otimes\left[a_{0}\left|D_{R, L}^{(0)}\right\rangle\left\langle D_{R, L}^{(0)}\left|+a_{+}\right| D_{R, L}^{(+)}\right\rangle\left\langle D_{R, L}^{(+)}\right|\right]
$$

and eqs. (11)-(13) become

$$
\begin{aligned}
\mathrm{d}|\psi\rangle & =\left[\sqrt{\gamma}\left(\hat{A_{L}}-\left\langle\hat{A_{L}}\right\rangle_{\psi_{t}}\right) \mathrm{d} B_{L}-\frac{1}{2} \gamma\left(\hat{A_{L}}-\left\langle\hat{A_{L}}\right\rangle_{\psi_{t}}\right)^{2} \mathrm{~d} t\right. \\
& \left.+\sqrt{\gamma}\left(\hat{A_{R}}-\left\langle\hat{A_{R}}\right\rangle_{\psi_{t}}\right) \mathrm{d} B_{R}-\frac{1}{2} \gamma\left(\hat{A_{R}}-\left\langle\hat{A_{R}}\right\rangle_{\psi_{t}}\right)^{2} \mathrm{~d} t\right]|\psi\rangle, \\
\overline{\mathrm{d} B_{R, L}} & =0, \quad \overline{\left(\mathrm{d} B_{R, L}\right)^{2}}=\mathrm{d} t, \quad \overline{\mathrm{d} B_{R} \mathrm{~d} B_{L}}=0, \\
\left\langle\hat{A_{R}}\right\rangle_{\psi_{t}} & =\left\langle\hat{A_{L}}\right\rangle_{\psi_{t}}=\langle\hat{A}\rangle_{\psi_{t}}=a_{0}\left|c_{L}\right|^{2}+a_{+}\left|c_{R}\right|^{2} .
\end{aligned}
$$

By following the same procedure sketched in the previous section, one obtains

$$
\begin{aligned}
L_{R, L}(t)=1+[ & \pm a \sqrt{\gamma}\left|c_{L, R}\right|^{2} \mathrm{~d} B_{R, L}-\frac{1}{2} \gamma a^{2}\left|c_{L, R}\right|^{4} \mathrm{~d} t \\
& \left.\mp a \sqrt{\gamma}\left|c_{L, R}\right|^{2} \mathrm{~d} B_{L, R}-\frac{1}{2} \gamma a^{2}\left|c_{L, R}\right|^{4} \mathrm{~d} t\right]
\end{aligned}
$$

from which

$$
L_{R, L}^{2}(t)=1 \pm 2 a \sqrt{\gamma}\left|c_{L, R}\right|^{2} \mathrm{~d} B_{R, L} \mp 2 a \sqrt{\gamma}\left|c_{L, R}\right|^{2} \mathrm{~d} B_{L, R}
$$

It is worth noticing that eq. (27) can be rewritten as

$$
L_{R, L}^{2}(t)=1 \pm 2 a \sqrt{\gamma}\left|c_{L, R}\right|^{2}\left(\mathrm{~d} B_{R}-\mathrm{d} B_{L}\right)=1 \pm 2 a \sqrt{\gamma}\left|c_{L, R}\right|^{2} \mathrm{~d} C
$$

where

$$
\mathrm{d} C=\mathrm{d} B_{R}-\mathrm{d} B_{L}
$$

is a gaussian stochastic variable obeying the statistics

$$
\overline{\mathrm{d} C}=0, \quad \overline{(\mathrm{d} C)^{2}}=\overline{\left(\mathrm{d} B_{R}\right)^{2}}+\overline{\left(\mathrm{d} B_{L}\right)^{2}}=2 \mathrm{~d} t .
$$

\section{THE SYSTEM/DETECTOR INTERACTION AND ITS INTERPLAY WITH THE STOCHASTIC PROCESS}

In the previous sections the system/apparatus interactions generating the superpositions (9) and (21) have been considered "instantaneous". Actually, any real device has a finite

reaction time $T$ and the dynamical development of the superpositions can be modelled by proper interaction hamiltonians (see for instance ref. [11], where the von Neumann model of 
measurement in quantum mechanics is described in detail, and ref. [1] for a recent discussion about the interplay between the measurement time and the localisation process).

Considering the single detector case, the system/apparatus interactions hamiltonian can be written as

$$
\hat{H}_{I}=\frac{\mathrm{d} \beta}{\mathrm{d} t} f(\hat{R}) \hat{P}
$$

where $\hat{P}$ is the momentum operator of the pointer, $R$ is defined as

$$
\hat{R}|\gamma\rangle_{R}=|\gamma\rangle_{R} \quad \hat{R}|\gamma\rangle_{L}=0
$$

the function $f$ satisfies

$$
f(0)=0, \quad f(1)=a,
$$

$\beta(t)$ is the activation function that, for the sake of simplicity, is assumed piecewise linear starting from $t=0$ taken as the interaction initial time

$$
\beta(t)=\min (t / T, 1) \quad t \geq 0
$$

and $T$ is the time at which the interaction has fully developed.

The effect of the interaction hamiltonian (31) on the initial superposition

$$
|\psi\rangle=\left(c_{L}|\gamma\rangle_{L}+c_{R}|\gamma\rangle_{R}\right)\left|D^{(0)}\right\rangle
$$

is the following: since $\hat{R}|\gamma\rangle_{L}=0$, the term $c_{L}|\gamma\rangle_{L}\left|D^{(0)}\right\rangle$ is left unchanged; on the contrary, since $\hat{R}|\gamma\rangle_{R}=|\gamma\rangle_{R}$, the pointer position in the term $c_{R}|\gamma\rangle_{R}\left|D^{(0)}\right\rangle$ is shifted from position "0" to position " $a$ ", corresponding to $c_{R}|\gamma\rangle_{R}\left|D^{(+)}\right\rangle$, linearly in the time interval $(t=0, t=T)$.

When taking into account the interaction (31), eq. (11) becomes

$$
\mathrm{d}|\psi\rangle=\left[-\frac{i}{\hbar} \hat{H}_{I} \mathrm{~d} t+\sqrt{\gamma}\left(\hat{A}-\langle\hat{A}\rangle_{\psi_{t}}\right) \mathrm{d} B-\frac{1}{2} \gamma\left(\hat{A}-\langle\hat{A}\rangle_{\psi_{t}}\right)^{2} \mathrm{~d} t\right]|\psi\rangle .
$$

Under the (very good) approximation that the stochastic process does not affect the pointer wave functions corresponding to different pointer positions, but affects only the coefficients of the superposition $\left(c_{R, L}\right)$, eq. (18) becomes

$$
L_{R, L}(t)=1+\left[ \pm a \sqrt{\gamma} \beta(t)\left|c_{L, R}\right|^{2} \mathrm{~d} B-\frac{1}{2} \gamma a^{2} \beta^{2}(t)\left|c_{L, R}\right|^{4} \mathrm{~d} t\right]
$$

from which

$$
L_{R, L}^{2}(t)=1 \pm 2 a \sqrt{\gamma} \beta(t)\left|c_{L, R}\right|^{2} \mathrm{~d} B
$$




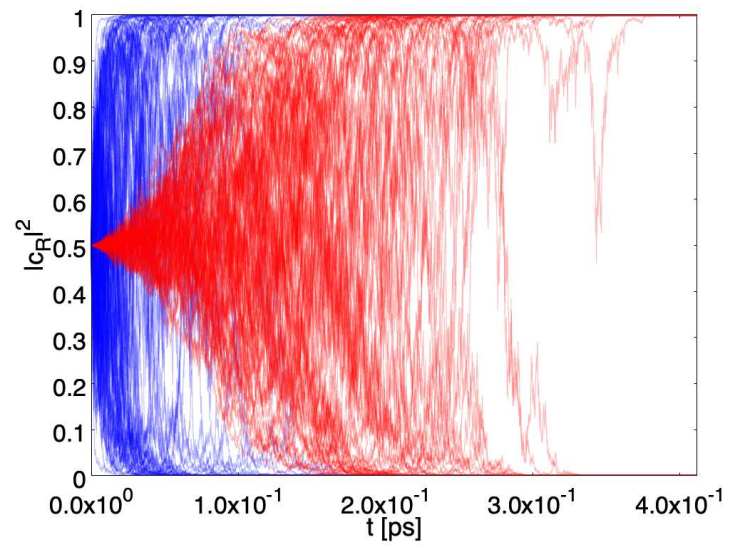

(a) Starting condition: $\left|c_{R}\right|^{2}=\frac{1}{2}$.

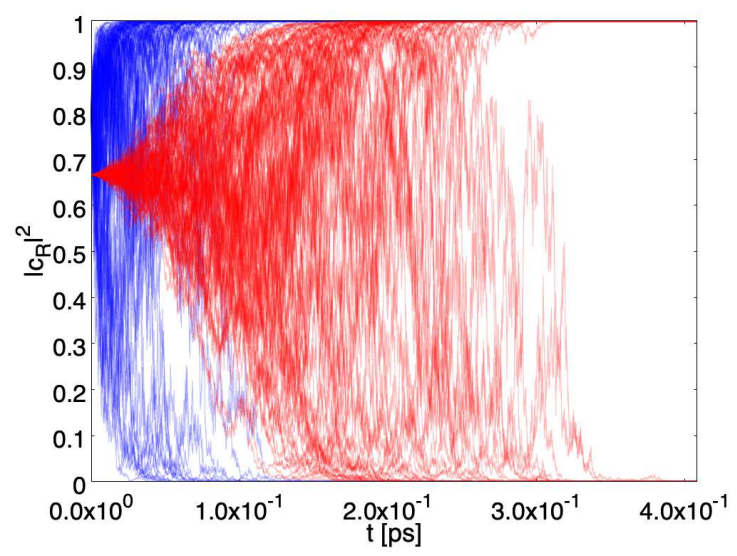

(c) Starting condition: $\left|c_{R}\right|^{2}=\frac{4}{5}$.

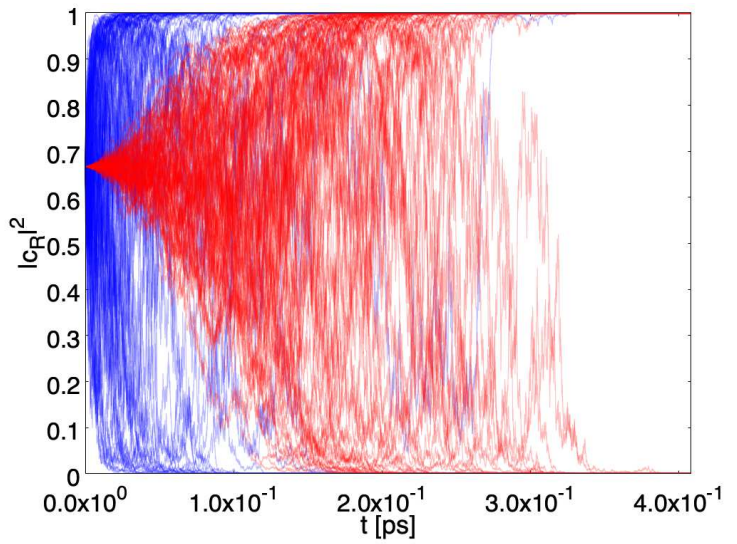

(b) Starting condition: $\left|c_{R}\right|^{2}=\frac{2}{3}$.

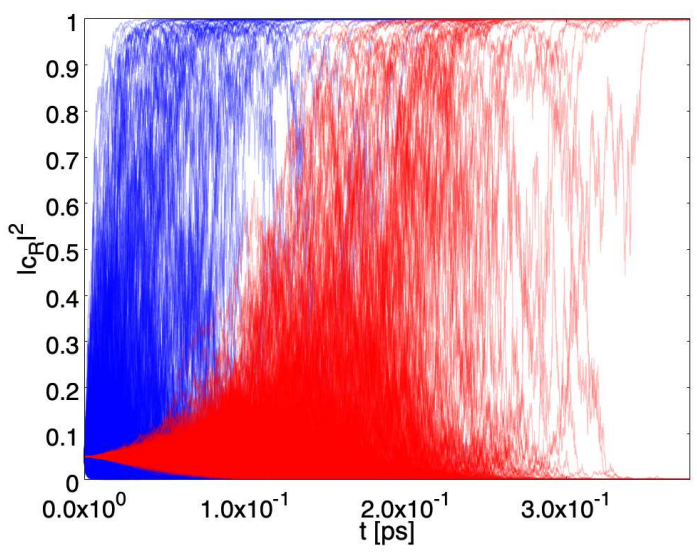

(d) Starting condition: $\left|c_{R}\right|^{2}=\frac{1}{20}$.

FIG. 3: Paths of $\left|c_{R}\right|^{2}$ as function of $t$ in case of a single detector with $\gamma=\gamma_{1}$. The time scale in ns $=10^{-9} \mathrm{~s}$. For all the figures, in blue are the paths for the case without the von Neumann activation (vNa), in red are the paths with vNa for $T=10^{-4} \mathrm{~ns}$.

and, analogously, eq. (26) turns into

$$
\begin{aligned}
L_{R, L}(t)=1+[ & \pm a \sqrt{\gamma} \beta(t)\left|c_{L, R}\right|^{2} \mathrm{~d} B_{R, L}-\frac{1}{2} \gamma a^{2} \beta^{2}(t)\left|c_{L, R}\right|^{4} \mathrm{~d} t \\
& \left.\mp a \sqrt{\gamma} \beta(t)\left|c_{L, R}\right|^{2} \mathrm{~d} B_{L, R}-\frac{1}{2} \gamma a^{2} \beta^{2}(t)\left|c_{L, R}\right|^{4} \mathrm{~d} t\right]
\end{aligned}
$$

from which

$$
\begin{aligned}
L_{R, L}^{2}(t) & =1 \pm 2 a \sqrt{\gamma} \beta(t)\left|c_{L, R}\right|^{2} \mathrm{~d} B_{R, L} \mp 2 a \sqrt{\gamma} \beta(t)\left|c_{L, R}\right|^{2} \mathrm{~d} B_{L, R} \\
& =1 \pm 2 a \sqrt{\gamma} \beta(t)\left|c_{L, R}\right|^{2} \mathrm{~d} C .
\end{aligned}
$$

It is worth noticing that the difference between eqs. (20) and (28) on one side, and eqs. (38) and (40) on the other, consists in the presence of the activation function $\beta(t)$ in the stochastic 


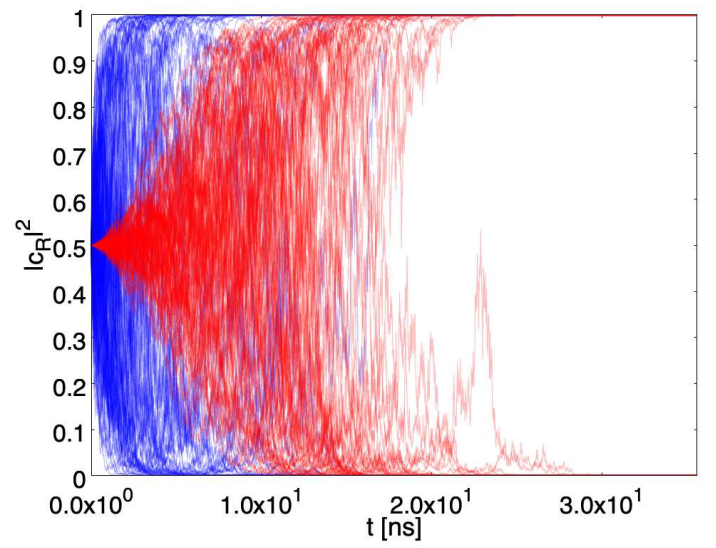

(a) Starting condition: $\left|c_{R}\right|^{2}=\frac{1}{2}$.

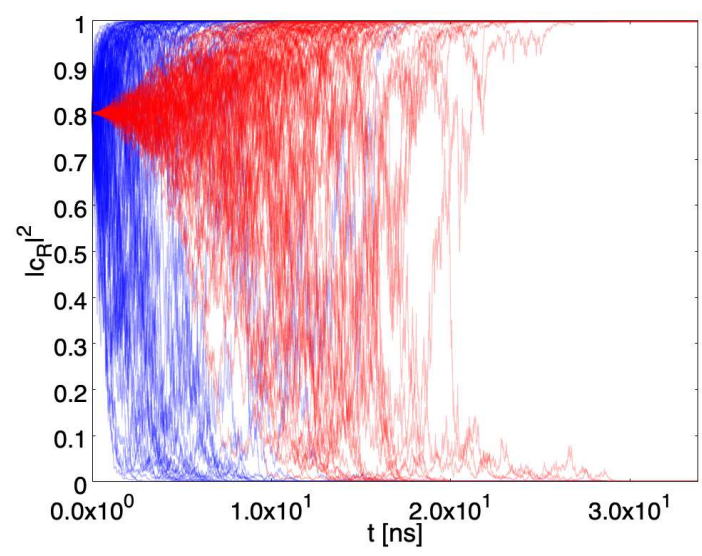

(c) Starting condition: $\left|c_{R}\right|^{2}=\frac{4}{5}$.

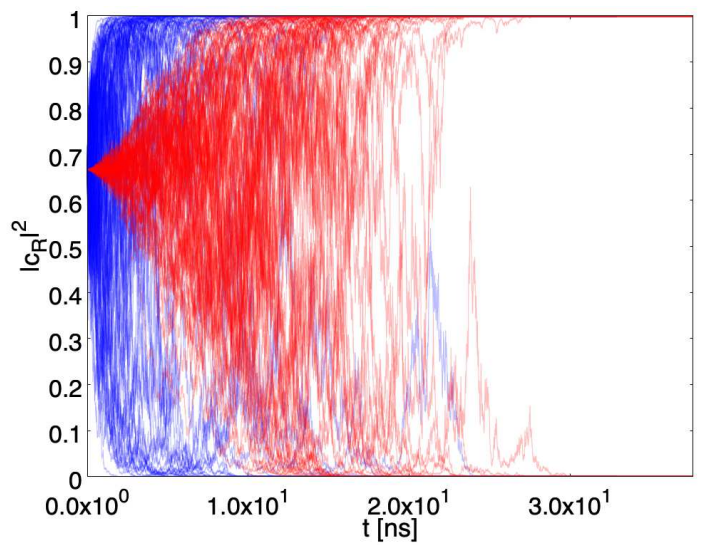

(b) Starting condition: $\left|c_{R}\right|^{2}=\frac{2}{3}$.

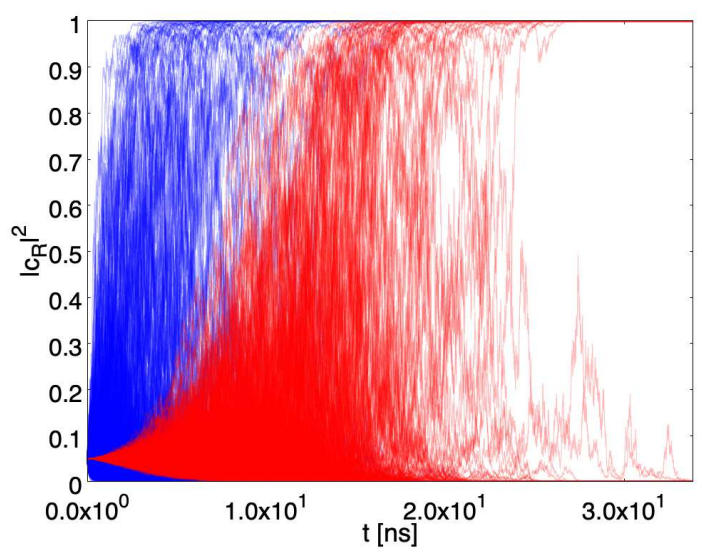

(d) Starting condition: $\left|c_{R}\right|^{2}=\frac{1}{20}$.

FIG. 4: Paths of $\left|c_{R}\right|^{2}$ as function of $t$ in case of a single detector with $\gamma=\gamma_{2}$. The time scale in $\mathrm{ns}=10^{-9} \mathrm{~s}$. For all the figures, in blue are the paths for the case without vNa, in red are the paths for $T=5 \mathrm{~ns}$.

term, and can be qualitatively understood as follows: the stochastic localization process is the more effective the more separate are the positions of the terms in the superposition. Since the interaction hamiltonian (31) generates the superposition gradually, the stochastic process starts at time $t=0$ and becomes fully effective at time $t=T$, when $\beta(T)=1$. 


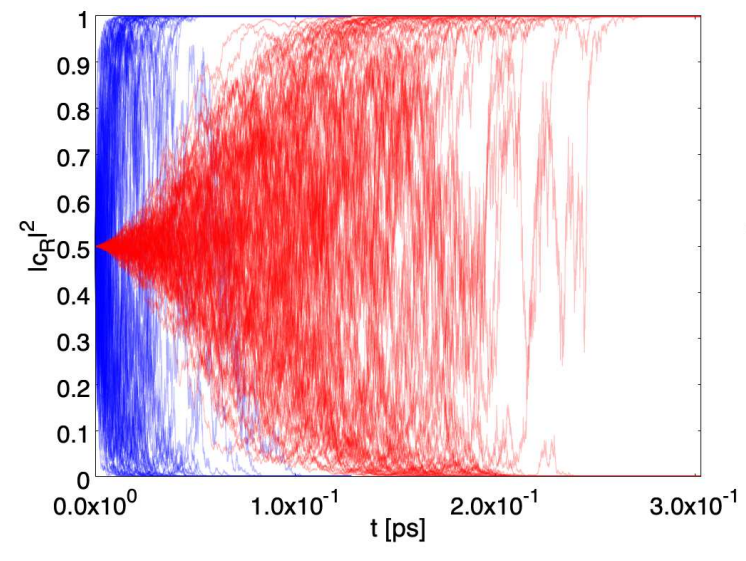

(a) Starting condition: $\left|c_{R}\right|^{2}=\frac{1}{2}$.

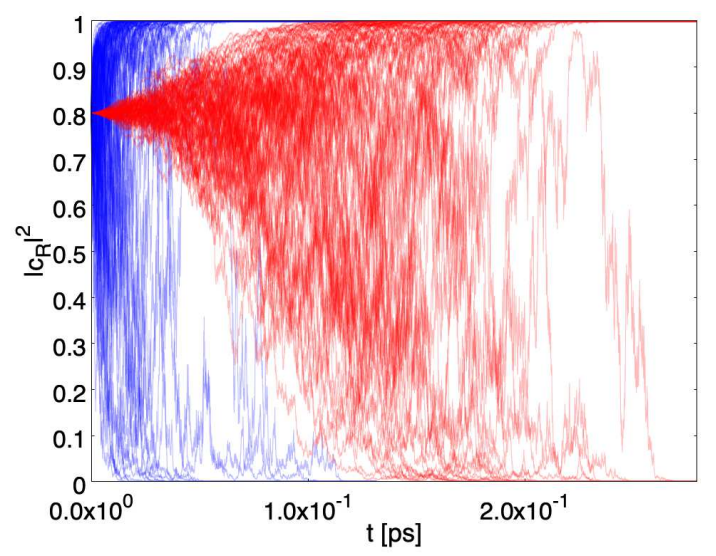

(c) Starting condition: $\left|c_{R}\right|^{2}=\frac{4}{5}$.

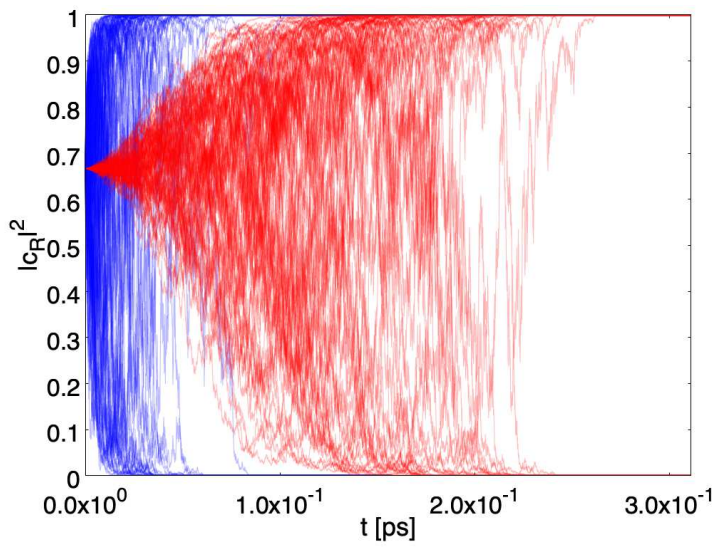

(b) Starting condition: $\left|c_{R}\right|^{2}=\frac{2}{3}$.

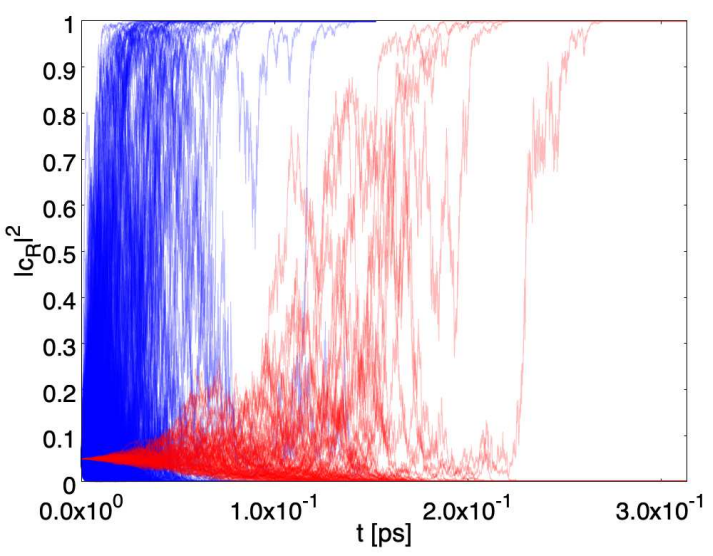

(d) Starting condition: $\left|c_{R}\right|^{2}=\frac{1}{20}$.

FIG. 5: Paths of $\left|c_{R}\right|^{2}$ as function of $t$ in case of two detectors with $\gamma=\gamma_{1}$. The time scale in ns $=10^{-9} \mathrm{~s}$. For all the figures, in blue are the paths for the case without vNa, in red are the paths for $T_{\mathrm{vNa}}=10^{-4} \mathrm{~ns}$.

\section{NUMERICAL RESULTS}

The original choice of parameters, for a process of localisation in position space, in [8] was

$$
\lambda_{\text {micro }}=10^{-16} \mathrm{~s}^{-1} \quad \alpha^{-1 / 2}=10^{-5} \mathrm{~cm}
$$

Recently, the experimental search of spontaneous X-ray emission [13] has possibly excluded this set of paramenters. A possible choice compatible with [13] is

$$
\lambda_{\text {micro }}=10^{-17} \mathrm{~s}^{-1} \quad \alpha^{-1 / 2}=10^{-4} \mathrm{~cm},
$$


that, according to eq. (8), corresponds to

$$
\gamma_{\text {micro }}=\frac{1}{2} 10^{-9} \mathrm{~cm}^{-2} \mathrm{~s}^{-1}
$$

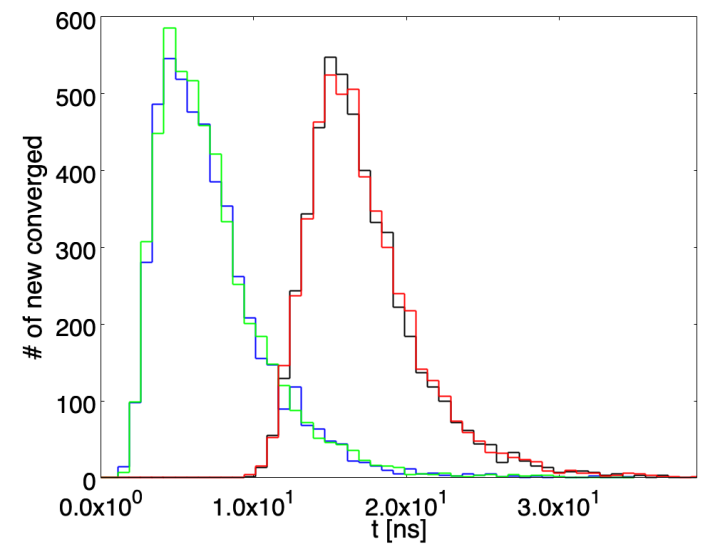

(a) Starting condition: $\left|c_{R}\right|^{2}=\frac{1}{2}$.

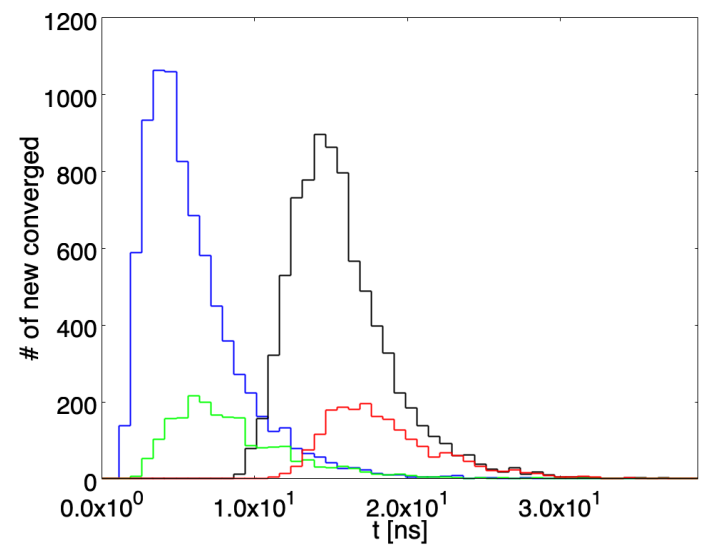

(c) Starting condition: $\left|c_{R}\right|^{2}=\frac{4}{5}$.

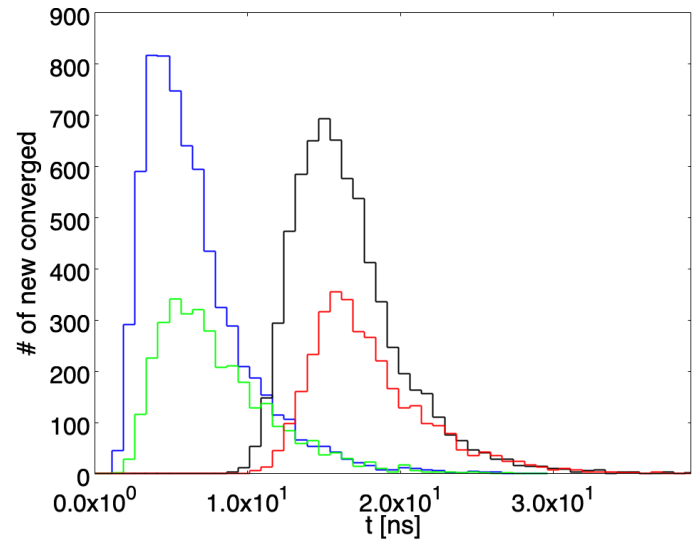

(b) Starting condition: $\left|c_{R}\right|^{2}=\frac{2}{3}$.

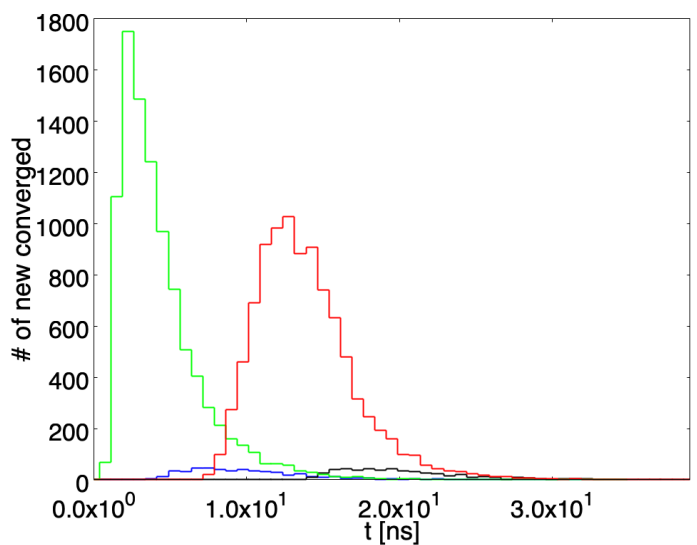

(d) Starting condition: $\left|c_{R}\right|^{2}=\frac{1}{20}$.

FIG. 6: The number of paths that, at each time bin, reached $\left|c_{R}\right|^{2} \geq 1-\varepsilon$ (blue and black histograms for vNa switched off/on, respetively) or $\left|c_{R}\right|^{2} \leq \varepsilon$ (green and red histograms for vNa switched off/on, respectively), with $\varepsilon=1 / N, N$ being the number of paths considered in the statistical sample $\left(10^{3}\right.$ in this case), for a single detector and $\gamma=\gamma_{2}$. The integral of any single histogram represents the total number of paths arrived at convergence (Born Rule).

For an object of approximately $1 \mathrm{~cm}^{3}$ the number of elementary constituents is assumed to be $10^{23}$. Consequently, if the dimension of the object is reduced to $1 \mathrm{~mm}^{3}$ one has 
approximately $10^{20}$ constituents. From these considerations, in the first case one has

$$
\gamma_{\text {macro }, 1} \equiv \gamma_{1}=10^{23} \gamma_{\text {micro }}=\frac{1}{2} 10^{14} \mathrm{~cm}^{-2} \mathrm{~s}^{-1}
$$

and in the second case

$$
\gamma_{\text {macro }, 2} \equiv \gamma_{2}=10^{20} \gamma_{\text {micro }}=\frac{1}{2} 10^{11} \mathrm{~cm}^{-2} \mathrm{~s}^{-1}
$$

Correspondingly, it is assumed that the parameter a describilng the "pointer" shift in position is $a=1 \mathrm{~cm}$ in the first case and $a=1 \mathrm{~mm}$ in the second one.

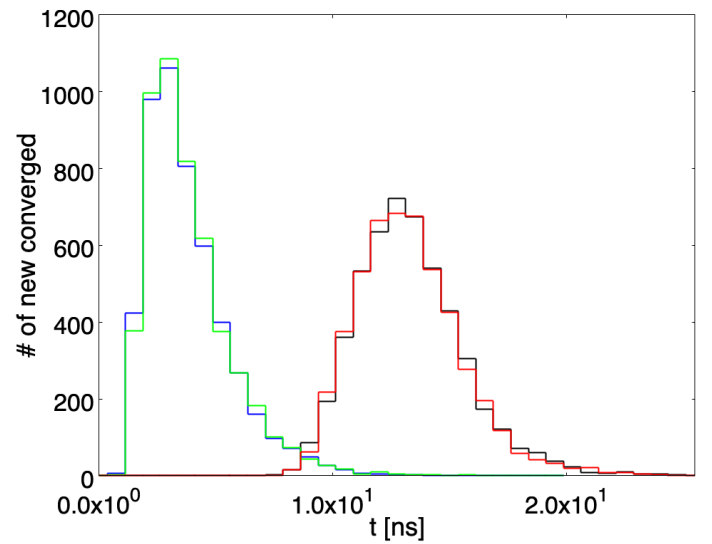

(a) Starting condition: $\left|c_{R}\right|^{2}=\frac{1}{2}$.

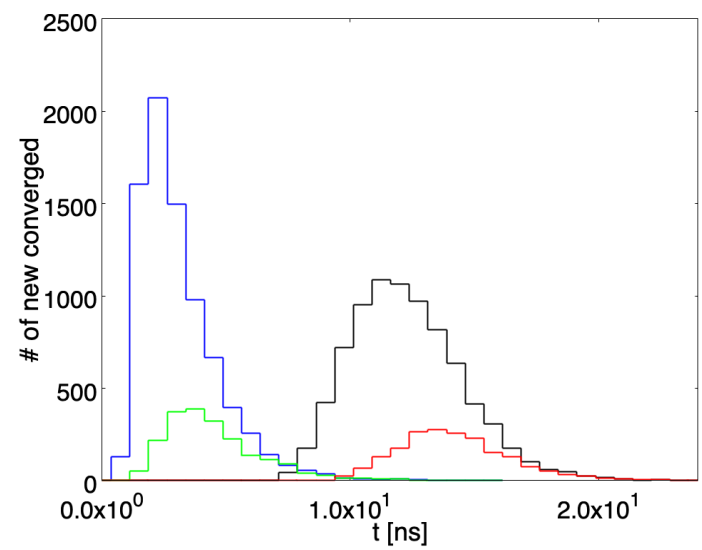

(c) Starting condition: $\left|c_{R}\right|^{2}=\frac{4}{5}$.

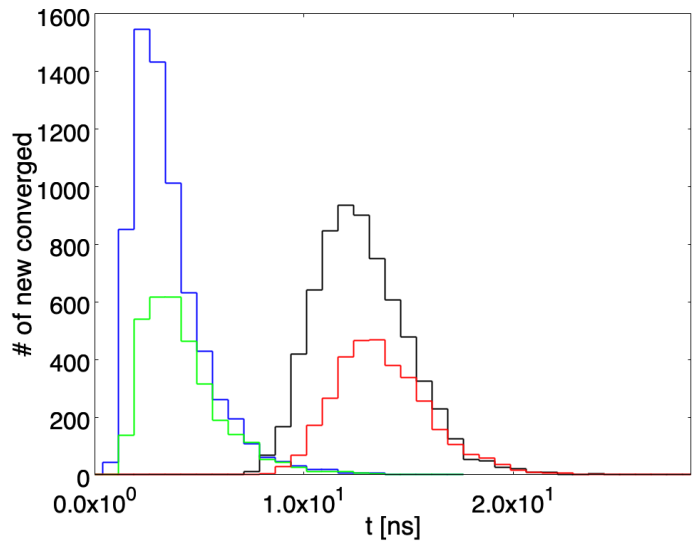

(b) Starting condition: $\left|c_{R}\right|^{2}=\frac{2}{3}$.

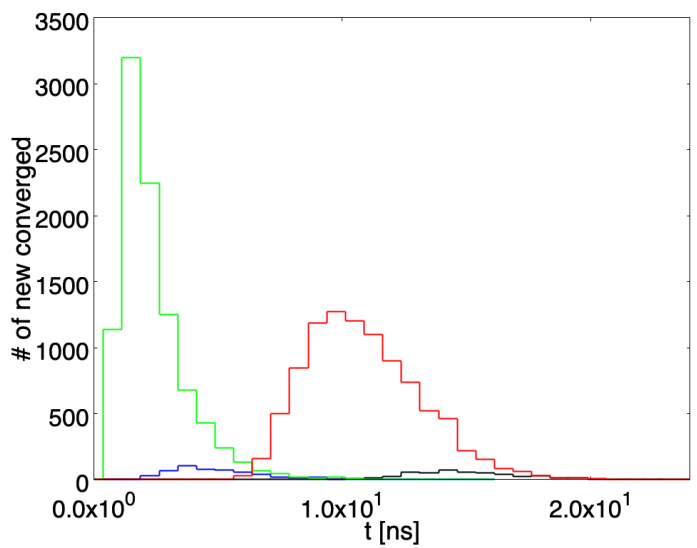

(d) Starting condition: $\left|c_{R}\right|^{2}=\frac{1}{20}$.

FIG. 7: The same as in Figure 6 for the two-detectors case.

Figure 3 shows a sample of $10^{3}$ paths followed by $\left|c_{R}(t)\right|^{2}$ for the starting conditions $\left|c_{R}\right|^{2}=1 / 2,2 / 3,4 / 5$ and $1 / 20$ for the case of a single detector with $\gamma=\gamma_{1}$. The blue/red paths correspond to the effect of the stochastic process alone and to the combined effect 
of stochastic process and von Neumann activation (vNa) with $T=10^{-4} \mathrm{~ns}$, respectively. The paths tend to converge to $\left|c_{R}\right|^{2}=1$ or $\left|c_{R}\right|^{2}=0$ with probability given by the starting condition (Born rule). For the stochastic process alone, the time scale at which the paths approach convergence is of the order of $5 \times 10^{-5} \mathrm{~ns}$, while in the presence of vNa the convergence is more distributed in time and becomes clear for $t \simeq T$, as expected.

Figure 4 is the same as Figure 3, but for $\gamma=\gamma_{2}$ and $T=5$ ns. Now the time scale at which the paths approach convergence is of the order of $3 \mathrm{~ns}$ (no vNa). The lengthening of the convergence time is due to the fact that in this case the detector is smaller and hence fewer constituents contribute to the spontaneous collapse.

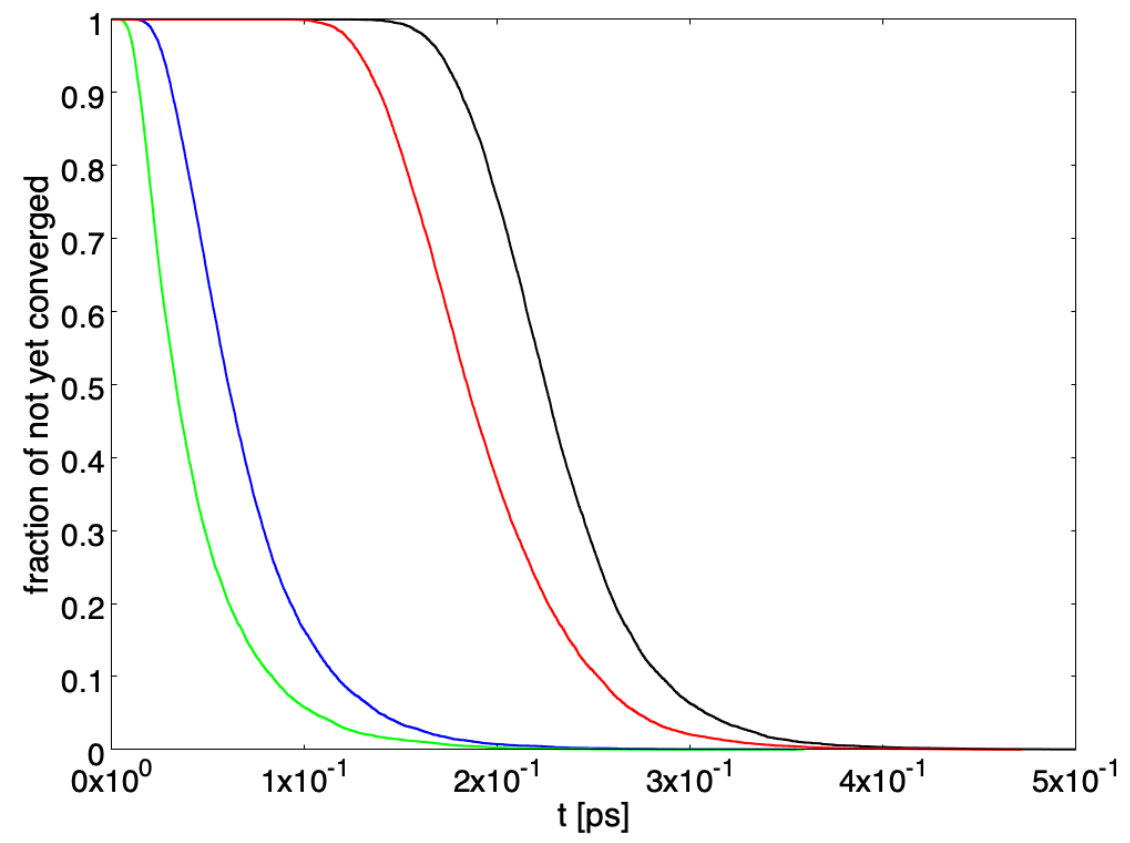

FIG. 8: Persistence of the superposition. Fraction of paths with $\varepsilon \leq\left|c_{R}\right|^{2} \leq 1-\varepsilon$. The green and blue curves correspond to the starting conditions $\left|c_{R}\right|^{2}=1 / 20$ and $1 / 2$, respectively, without vNa. The red and black curves represent the same situation with vNa. The setup with $\gamma=\gamma_{1}$ and a single detector has been chosen.

Figure 5 is the same as Figure 3, but for the case of two detectors. As can be seen, the convergence is more rapid than in the case of a single detector. The larger effectiveness of the localization process can be traced back to the statistical properties of the stochastic process $\mathrm{d} C$, whose variance is two times the variance of the individual stochastic processes $\mathrm{d} B$. 
In all these cases $T$ has been chosen larger than the typical convergence time scale; for $T$ less then, or similar to, the convergence time scale the stochastic process is not affected in a significant way.

In order to better quantify the convergence properties of the processes, Figure 6 shows the number of paths that, at each time bin, reached $\left|c_{R}\right|^{2} \geq 1-\varepsilon$ (blue and black histograms for vNa switched off/on, respetively) or $\left|c_{R}\right|^{2} \leq \varepsilon$ (green and red histograms for vNa switched off/on, respetively), with $\varepsilon=1 / N, N$ being the number of paths considered in the statistical sample, for the case of a single detector and $\gamma=\gamma_{2}$. The integral of any single histogram represents the total number of paths arrived at convergence (Born Rule). As can be noticed by comparing same color histograms, their shape and peak position depend on the starting conditions. For instance, looking at the blue histograms (paths converging to $\left|c_{R}\right|^{2}=1$ ), the peak present for the starting condition $\left|c_{R}\right|^{2}=4 / 5$ tends to flatten as the initial value for $\left|c_{R}\right|^{2}$ is reduced and its position shifts from about 5 to about $8 \mathrm{~ns}$ for the starting condition $\left|c_{R}\right|^{2}=1 / 20$. Qualitatively similar comments hold also for Figure 7, where the case of two detectors is shown.

Figure 8 shows the persistence of the superposition of $\left|\psi_{R, L}\right\rangle$ states. The fraction of paths with $\varepsilon \leq\left|c_{R}\right|^{2} \leq 1-\varepsilon$ is represented as a function of time. The green and blue curves correspond to the starting conditions $\left|c_{R}\right|^{2}=1 / 20$ and $1 / 2$, respectively, without vNa. The red and black curves represent the same situation with vNa. The setup with $\gamma=\gamma_{1}$ and a single detector has been chosen. As can be seen, both with and without vNa the starting condition $\left|c_{R}\right|^{2}=1 / 2$ corresponds to a longer lasting superposition, while with $\left|c_{R}\right|^{2}=1 / 20$ the superposition decays more rapidly. A qualitatively similar situation is found for the other setup previously considered.

At last, Figure 9 shows the persistence of the superposition for the paths converging to $\left|c_{R}\right|^{2}=1$. The fraction of paths converging to $\left|c_{R}\right|^{2}=1$ with $\varepsilon \leq\left|c_{R}\right|^{2} \leq 1-\varepsilon$ is represented as a function of time, The blue and green curves correspond to the starting conditions $\left|c_{R}\right|^{2}=1 / 2$ and $1 / 20$, respectively, without vNa. The black and red curves represent the same situation with vNa. The setup with $\gamma=\gamma_{1}$ and a single detector has been chosen. As can be seen, both with and without $\mathrm{vNa}$, for the paths converging to $\left|c_{R}\right|^{2}=1$ the superposition of $\left|\psi_{R, L}\right\rangle$ states is more persistent for the starting condition $\left|c_{R}\right|^{2}=1 / 20$ than for $\left|c_{R}\right|^{2}=1 / 2$. 


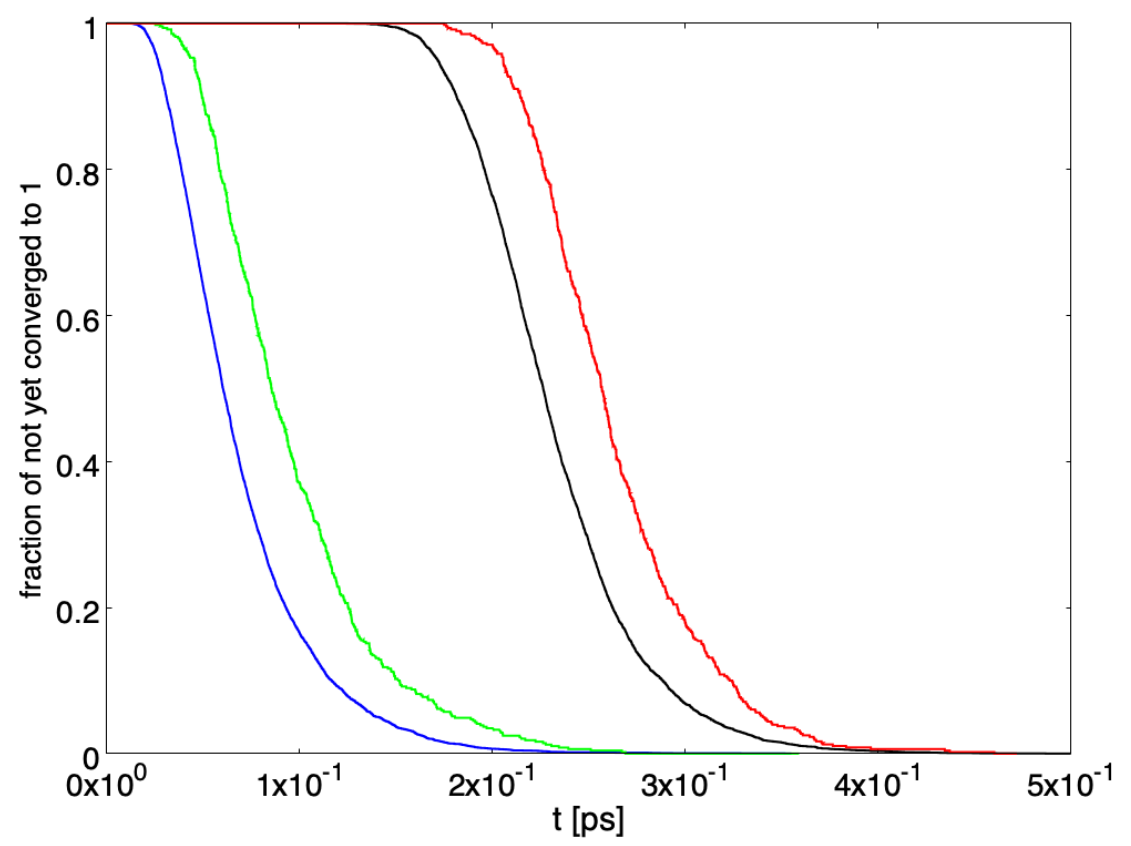

FIG. 9: Persistence of the superposition. Fraction of paths with $\varepsilon \leq\left|c_{R}\right|^{2} \leq 1-\varepsilon$ as a function of time, for the paths converging to $\left|c_{R}\right|^{2}=1$. The blue and green curves correspond to the starting conditions $\left|c_{R}\right|^{2}=1 / 2$ and $1 / 20$, respectively, without vNa. The black and red curves represent the same situation with vNa. The setup with $\gamma=\gamma_{1}$ and a single detector has been chosen.

\section{CONCLUSIONS}

In the present paper a detailed study of collapse dynamics as foreseen by GRW theory and its continuous realisations in the form of stochastic differential equations describing a brownian motion in the Hilbert space has been presented. The possible effect of the finite reaction time of the apparatus has also been considered. It has been assumed that a "pointer" shifts its position by an amount $a$ as a consequence of its measurement; this, of course, is not the general case, and what is the "pointer" has to be established in relation to the real physical detection and logging apparatus employed. The features of the convergence properties depend on the starting condition, and could be exploited to design experiments aiming at pointing out "GRW" effects.

The two elements determining the time resolution in the experiment outlined in figure 1 or 2 are the single photon source and the detectors. We will now briefly consider the 
challenges each one presents to the possibility of a an actual implementation of the proposed experiment.

A wide variety of single photon sources have been developed over the years, especially thanks to their applied potential in quantum technologies such as quantum communication and quantum computing. Among these sources, the most commonly used in quantum optical experiments fall in two categories: heralded single photon sources and quantum dots. In the first type of source optical nonlinearities are exploited to create photon pairs, followed by the detection of one of the photons and the corresponding projection of the second one on a single photon Fock state [4]. The advantages of these sources are that they can be very brilliant and, more importantly for the present experiment, the extraction efficiency of the photons from the source approaches unity. The main drawback of these sources is that they are probabilistic in nature, and there always exists a non-zero probability that multiple photon pairs are emitted at the same time, thus polluting the single photon state. The typical lifetime of photons generated with parametric sources can be below one picosecond.

In the case of quantum dots, the emission of a stream of single photons is granted by the Fermionic repulsion of electrons confined in quasi 0-dimensional nanostructures [2], often embedded in a semiconductor substrate. There are two main disadvantages of quantum dots; the first is that they generally operate at temperatures of the order of a few Kelvins, the second is that the extraction efficiency from the source is usually less than $50 \%$. This second issue is of particular importance for the proposed experiment; indeed the majority of the photons are generally scattered inside the semiconductor containing the quantum dot, to be quickly absorbed by the substrate. This high probability of absorption before reaching the detectors could result in a change in the collapse dynamics, hindering the experiment. The typical lifetime of photons generated by quantum dots is of the order of tens to hundreds of picoseconds.

Concerning the detectors, the most performant existing single photon detectors at optical and near infrared frequencies are Superconducting Single Photon Detectors (SSPDs) [17]. These devices consist in superconducting wires driven near to the critical current of the superconducting material. If properly designed, the energy of even a single photon absorbed by the wire is sufficient to deposit enough heat to break the superconducting state, thus generating a voltage spike. The time resolution of SSPDs is of the order of a few tens of picoseconds, and their quantum efficiencies are close to unity, generally larger than $95 \%$. 
The voltage spike generated by a detection event in SSPDs is then electronically amplified giving rise to electrical pulses. For a general set-up, we can assume such pulses to be $10 \mathrm{~ns}$ in time width and $10 \mathrm{~V}$ in amplitude. If the circuit is closed on a $50 \mathrm{Ohm}$ load, each pulse carries a charge of approximatively $10^{10}$ electrons, charge that is provided by capacitive elements within the amplifier circuit (the circuit then needs time to recharge, the so called "dead time" of the detectors).

If we assume the electronic pulse following detection events to be the "pointer", and assume that the set of observables to be sharpened is given by mass densities as in the last realisation discussed in ref. [6], the value of the parameter $\gamma$ would be significantly smaller than $\gamma_{2}$, resulting in collapse times much longer than 1 ns. It seems therefore possible to build, with existing technologies, an experiment as that outlined in figure 1 or 2 in which the collapse dynamics is longer than the time resolution given by the photon lifetime and the resolution of the detectors (expected to be hundreds of ps at the most). Such an experiment remains however challenging, given in particular the requirement that the almost totality of photons must be succesfully routed to the detectors, to avoid the possibility of collapse due to absorption of the photons from the various objects constituting the experimental set-up. One promising route to minimize this problem might be the use of a fully integrated experiment, in which single photons generated by a quantum dot are not extracted from the semiconductor, but instead emitted in an optical waveguide fabricated in the semiconductor itself. This process can be engineered to have high efficiency thanks to the Purcell effect [10]. The photons could then be routed toward monolithically integrated SSPDs. Recent experimental results [15] again show that such a goal can be considered within reach of existing photonic technologies.

Albeit the qualitative features of collapse dynamics, as previously shown, do not depend of the value of the parameter $\gamma$, collapse times are sensitive to the details of the particular detector employed. A tailored analysis is then required, taking into account all the particular aspects of the experimental setup adopted, and is left to future investigation. 
[1] Adler, S.L., Bassi, A., Ferialdi, L.: Minimum measurement time: lower bound on the frequency cutoff for collapse models. Journal of Physics A: Mathematical and Theoretical 53(21), 215302 (2020). doi:10.1088/1751-8121/ab8673. URL http://dx.doi.org/10.1088/ $1751-8121 / \mathrm{ab} 8673$

[2] Arakawa, Y., Holmes, M.J.: Progress in quantum-dot single photon sources for quantum information technologies: A broad spectrum overview. Applied Physics Reviews 7(2), 021309 (2020). doi:10.1063/5.0010193. URL https://doi.org/10.1063/5.0010193

[3] Bassi, A., Lochan, K., Satin, S., Singh, T.P., Ulbricht, H.: Models of Wave-function Collapse, Underlying Theories, and Experimental Tests. Rev. Mod. Phys. 85, 471-527 (2013). doi: 10.1103/RevModPhys.85.471

[4] Castelletto, S.A., Scholten, R.E.: Heralded single photon sources: a route towards quantum communication technology and photon standards. The European Physical Journal Applied Physics 41(3), 181-194 (2008). doi:10.1051/epjap:2008029. URL https : //doi .org/10.1051/ epjap: 2008029

[5] Diósi, L.: Continuous quantum measurement and itô formalism. Physics Letters A 129(8), 419-423 (1988). doi:https://doi.org/10.1016/0375-9601(88)90309-X. URL https://www . sciencedirect.com/science/article/pii/037596018890309X

[6] Ghirardi, G.C., Nicrosini, O., Rimini, A.: What really matters in hilbert-space stochastic processes. In: S. Gao (ed.) Collapse of the Wave Function: Models, Ontology, Origin, and Implications, chap. 2, pp. 12-22. Cambridge University Press (2018)

[7] Ghirardi, G.C., Pearle, P.M., Rimini, A.: Markov processes in Hilbert space and continuous spontaneous localization of systems of identical particles. Phys. Rev. A42, 78-79 (1990). doi:10.1103/PhysRevA.42.78

[8] Ghirardi, G.C., Rimini, A., Weber, T.: Unified dynamics for microscopic and macroscopic systems. Phys. Rev. D34, 470 (1986). doi:10.1103/PhysRevD.34.470

[9] Gisin, N.: Quantum measurements and stochastic processes. Phys. Rev. Lett. 52, 16571660 (1984). doi:10.1103/PhysRevLett.52.1657. URL https ://link . aps.org/doi/10.1103/ PhysRevLett.52.1657

[10] Liu, F., Brash, A.J., O’Hara, J., Martins, L.M.P.P., Phillips, C.L., Coles, R.J., Royall, B., 
Clarke, E., Bentham, C., Prtljaga, N., Itskevich, I.E., Wilson, L.R., Skolnick, M.S., Fox, A.M.: High purcell factor generation of indistinguishable on-chip single photons. Nature Nanotechnology 13(9), 835-840 (2018). doi:10.1038/s41565-018-0188-x. URL https://doi . org/10.1038/s41565-018-0188-x

[11] Mello, P., Johansen, L.: Measurements in quantum mechanics and von neumann's model. AIP Conference Proceedings 1319 (2010). doi:10.1063/1.3536611

[12] Nicrosini, O. and Rimini, A.: On the relationship between continuous and discontinuous stochastic processes in Hilbert space. Found. Phys. 20, 1317-1327 (1990)

[13] Piscicchia, K., Bassi, A., Curceanu, C., Grande, R., Donadi, S., Hiesmayr, B., Pichler, A.: Csl collapse model mapped with the spontaneous radiation. Entropy 19(7), 319 (2017). doi: 10.3390/e19070319. URL http://dx.doi.org/10.3390/e19070319

[14] Schlosshauer, M.: Decoherence, the measurement problem, and interpretations of quantum mechanics. Rev. Mod. Phys. 76, 1267-1305 (2005). doi:10.1103/RevModPhys.76.1267. URL https://link.aps.org/doi/10.1103/RevModPhys.76.1267

[15] Schwartz, M., Schmidt, E., Rengstl, U., Hornung, F., Hepp, S., Portalupi, S.L., llin, K., Jetter, M., Siegel, M., Michler, P.: Fully on-chip single-photon hanbury-brown and twiss experiment on a monolithic semiconductor-superconductor platform. Nano Letters 18(11), 6892-6897 (2018). doi:10.1021/acs.nanolett.8b02794. URL https://doi.org/10.1021/acs .nanolett. 8b02794

[16] Wechsler, S.: In praise and in criticism of the model of continuous spontaneous localization of the wave-function. arXiv: Quantum Physics (2020)

[17] Zhang, H., Xiao, L., Luo, B., Guo, J., Zhang, L., Xie, J.: The potential and challenges of time-resolved single-photon detection based on current-carrying superconducting nanowires. Journal of Physics D: Applied Physics 53(1), 013001 (2019). doi:10.1088/1361-6463/ab4146. URL https://doi.org/10.1088/1361-6463/ab4146 
Figures

\section{Undetected}

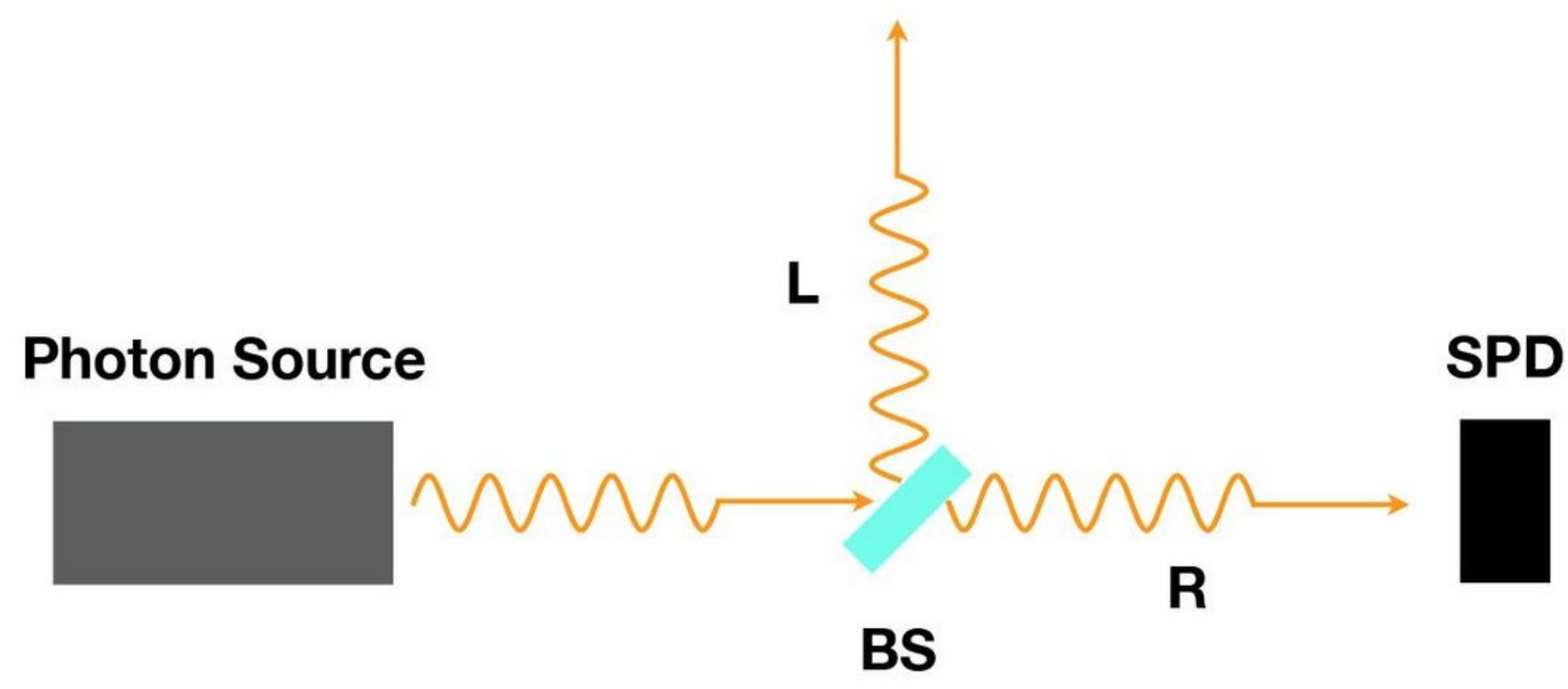

Figure 1

Please see the Manuscript PDF file for the complete figure caption 


\section{SPD}

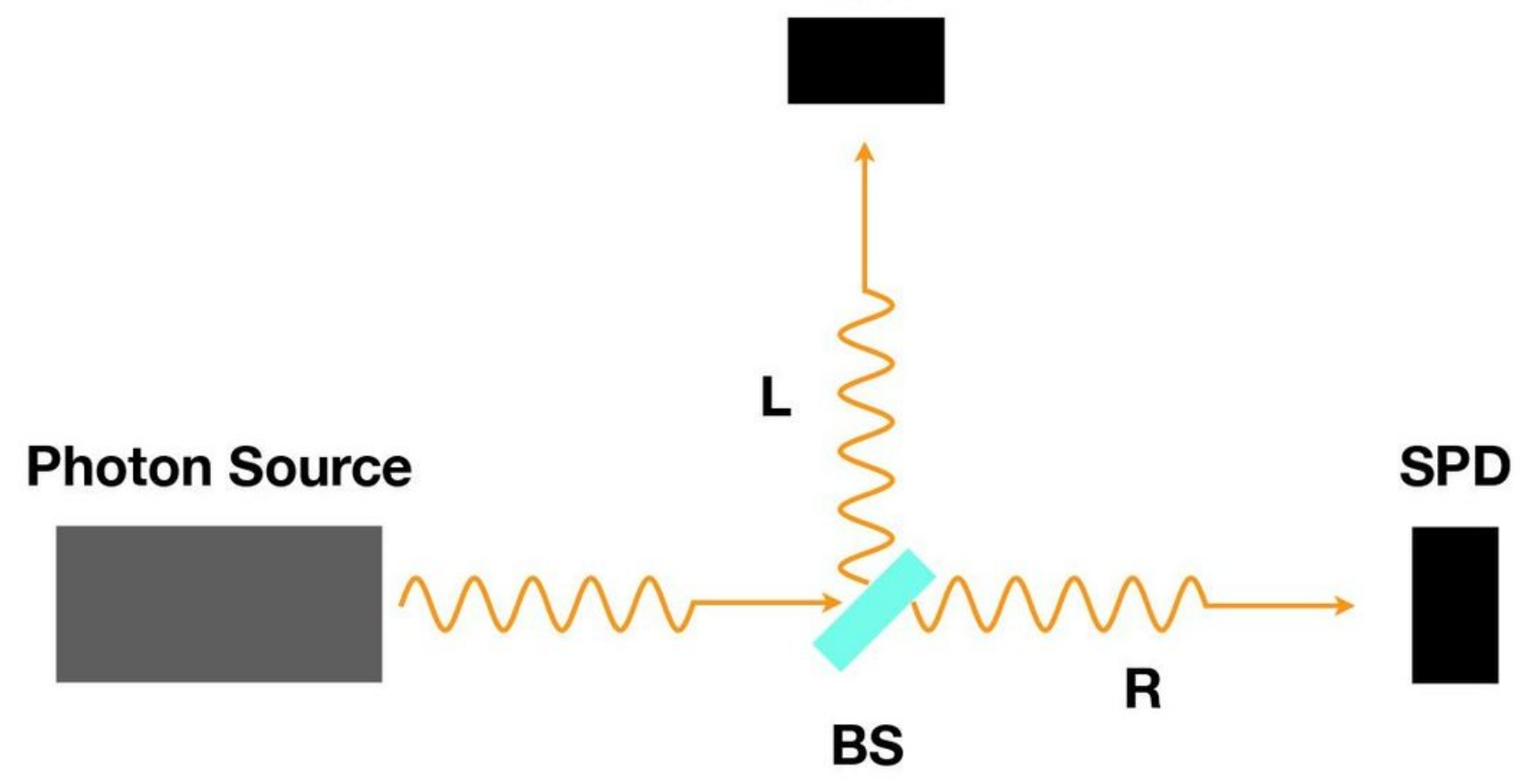

Figure 2

Please see the Manuscript PDF file for the complete figure caption 


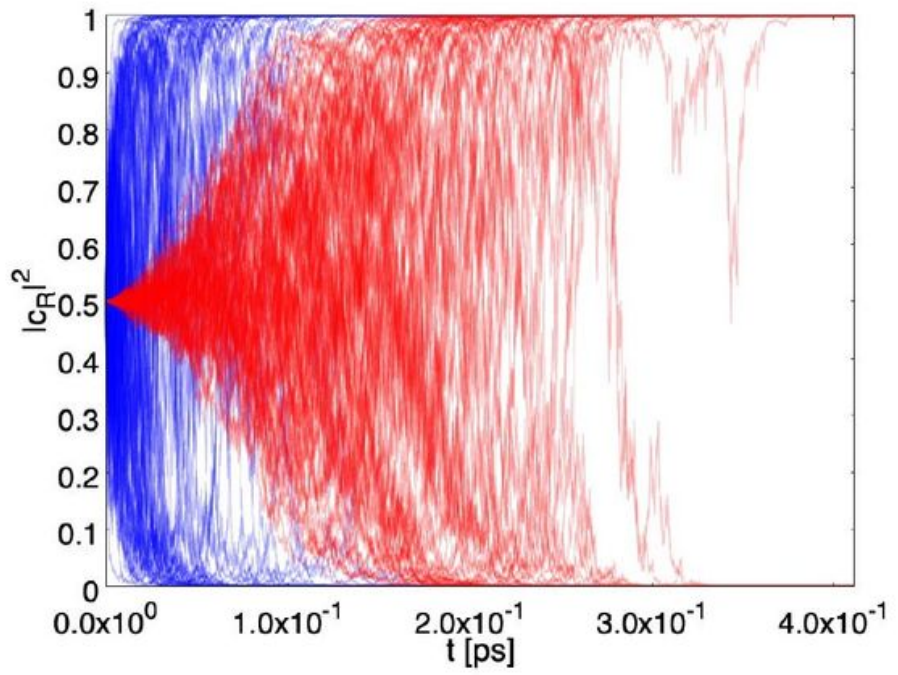

(a) Starting condition: $\left|c_{R}\right|^{2}=\frac{1}{2}$.

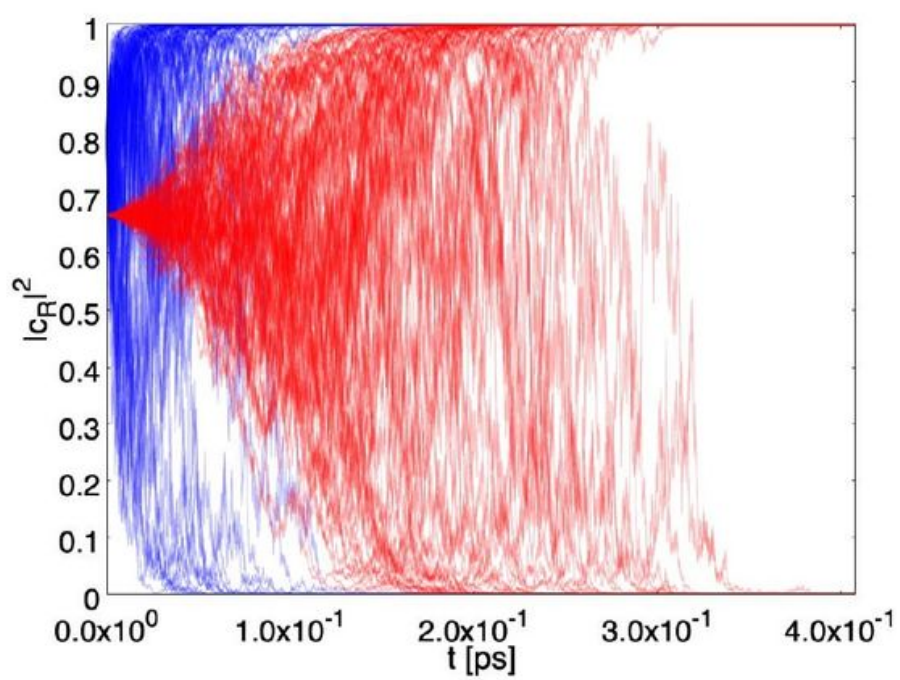

(c) Starting condition: $\left|c_{R}\right|^{2}=\frac{4}{5}$.

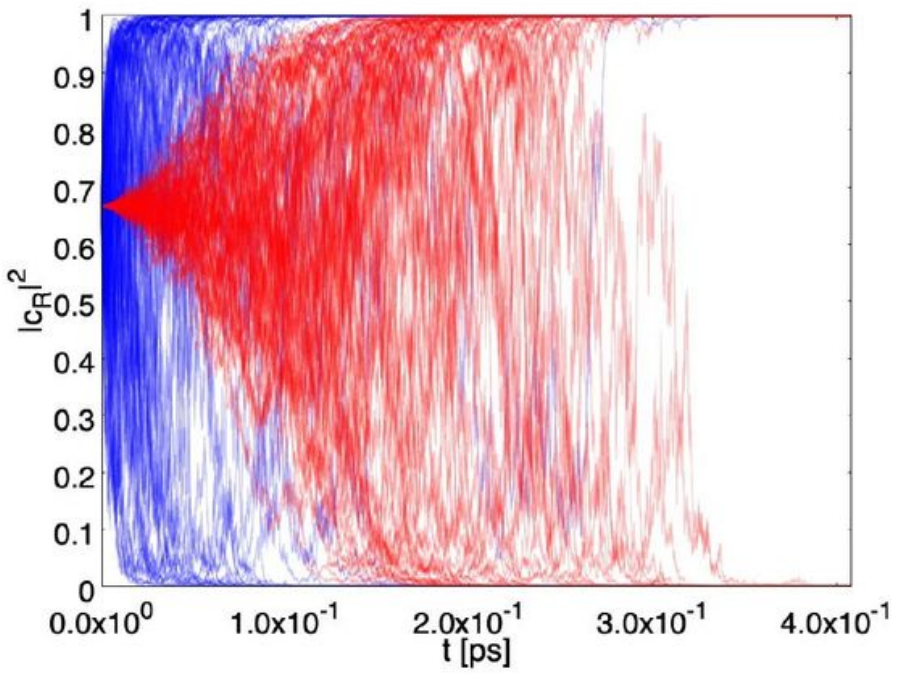

(b) Starting condition: $\left|c_{R}\right|^{2}=\frac{2}{3}$.

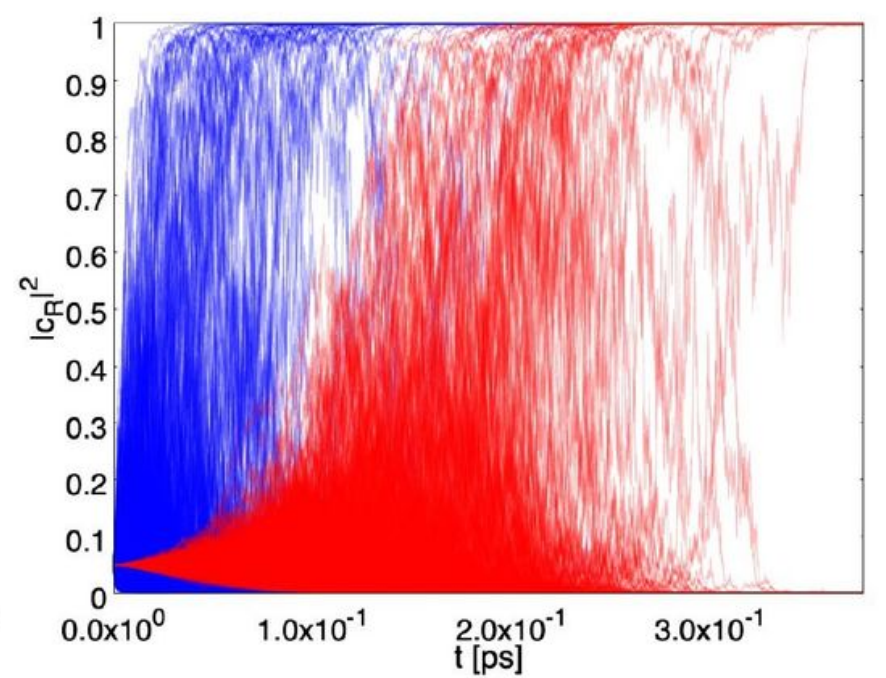

(d) Starting condition: $\left|c_{R}\right|^{2}=\frac{1}{20}$.

\section{Figure 3}

Please see the Manuscript PDF file for the complete figure caption 


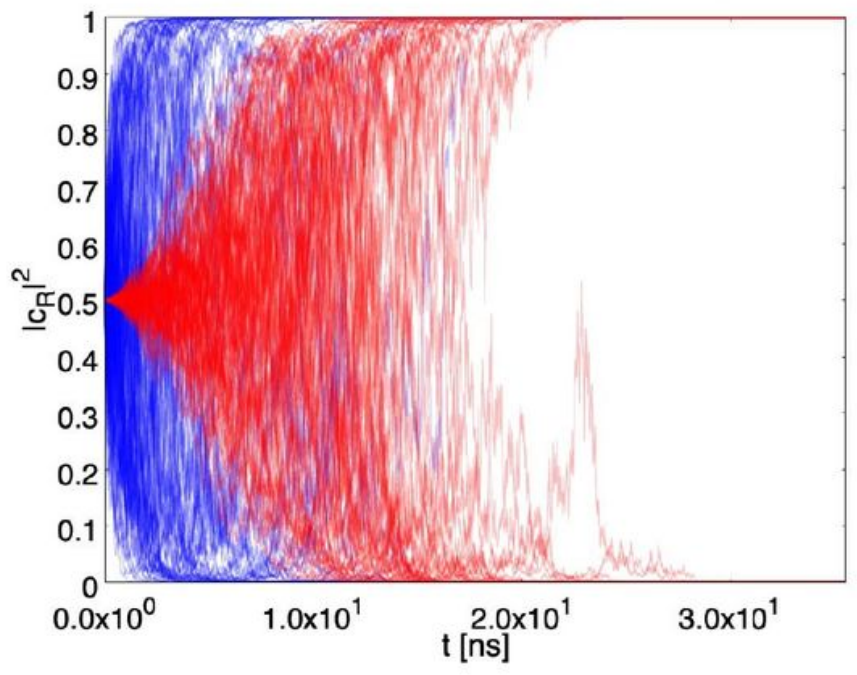

(a) Starting condition: $\left|c_{R}\right|^{2}=\frac{1}{2}$.

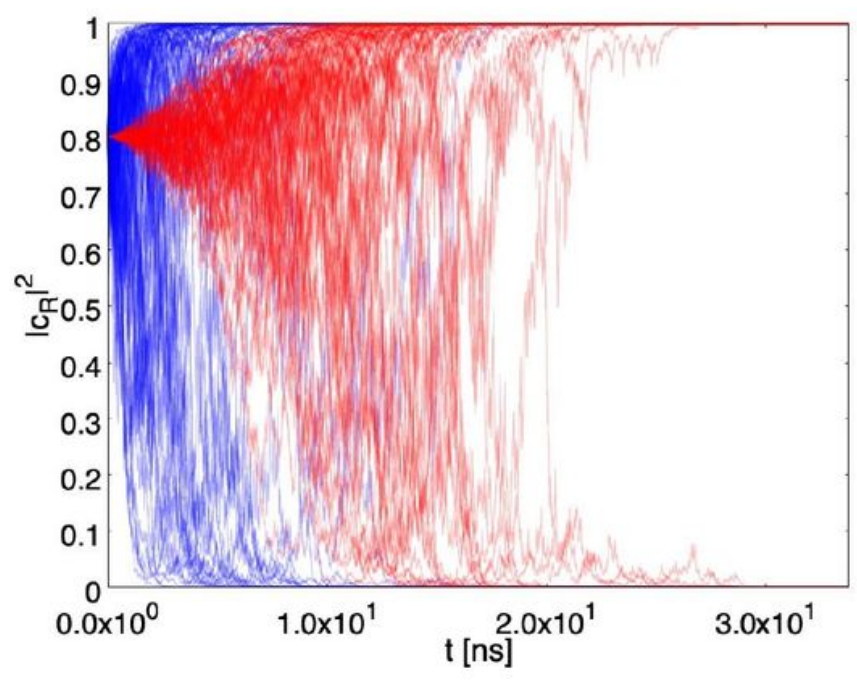

(c) Starting condition: $\left|c_{R}\right|^{2}=\frac{4}{5}$.

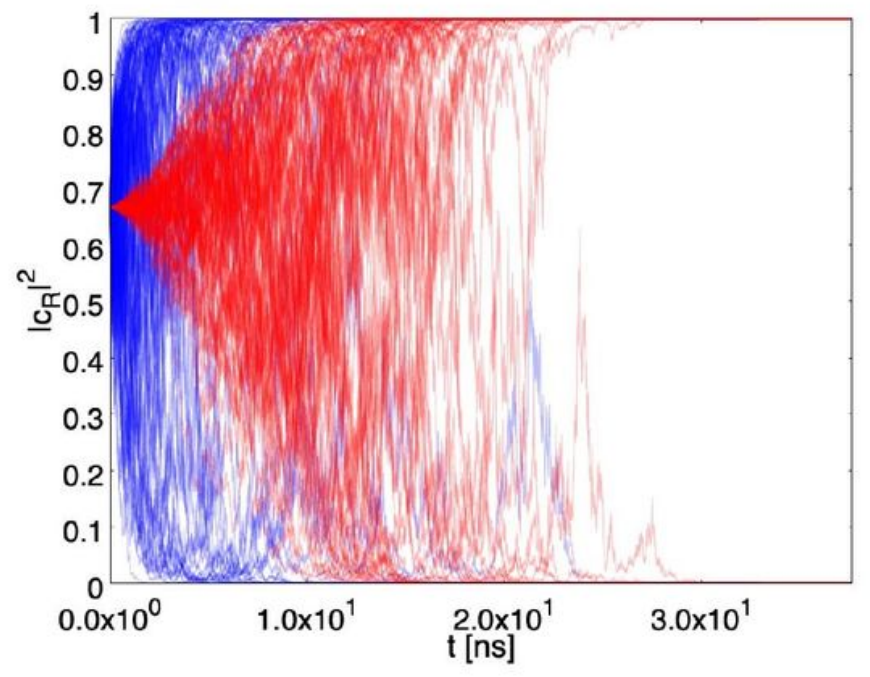

(b) Starting condition: $\left|c_{R}\right|^{2}=\frac{2}{3}$.

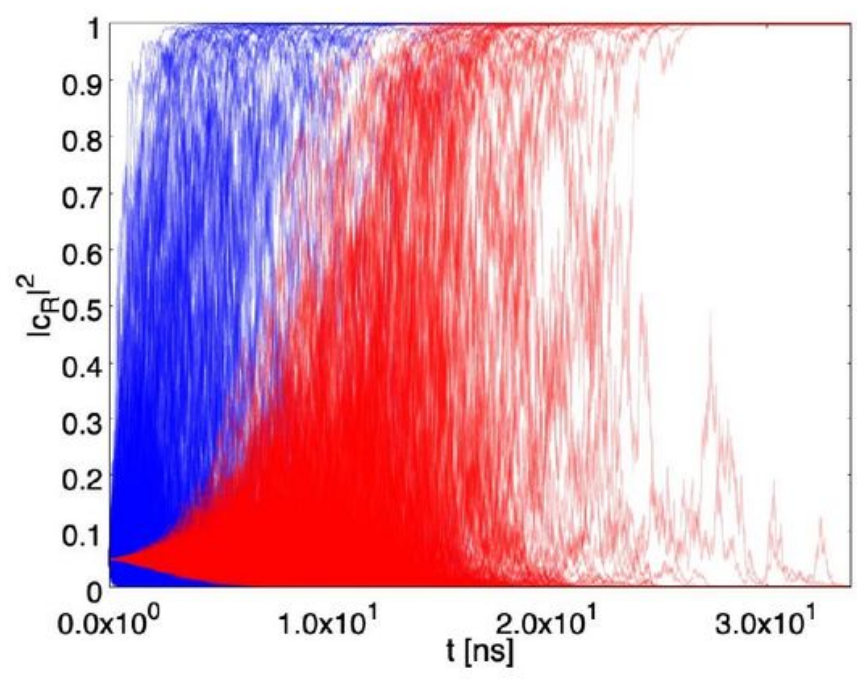

(d) Starting condition: $\left|c_{R}\right|^{2}=\frac{1}{20}$.

\section{Figure 4}

Please see the Manuscript PDF file for the complete figure caption 


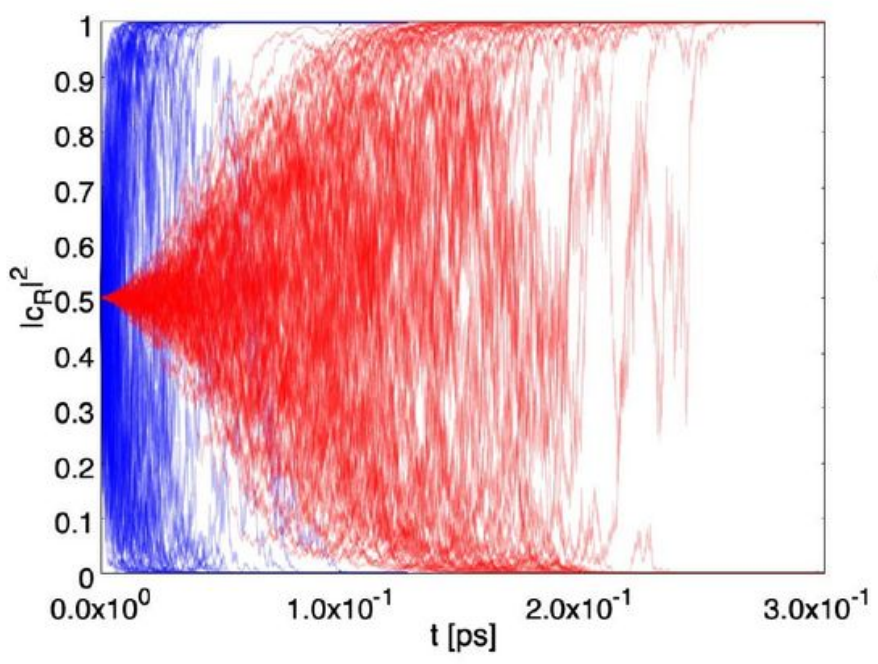

(a) Starting condition: $\left|c_{R}\right|^{2}=\frac{1}{2}$.

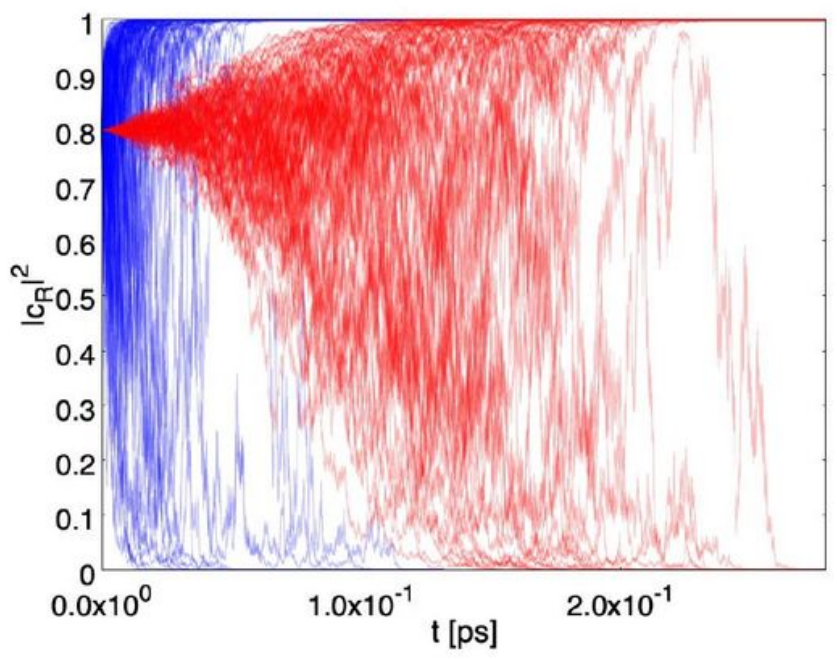

(c) Starting condition: $\left|c_{R}\right|^{2}=\frac{4}{5}$.

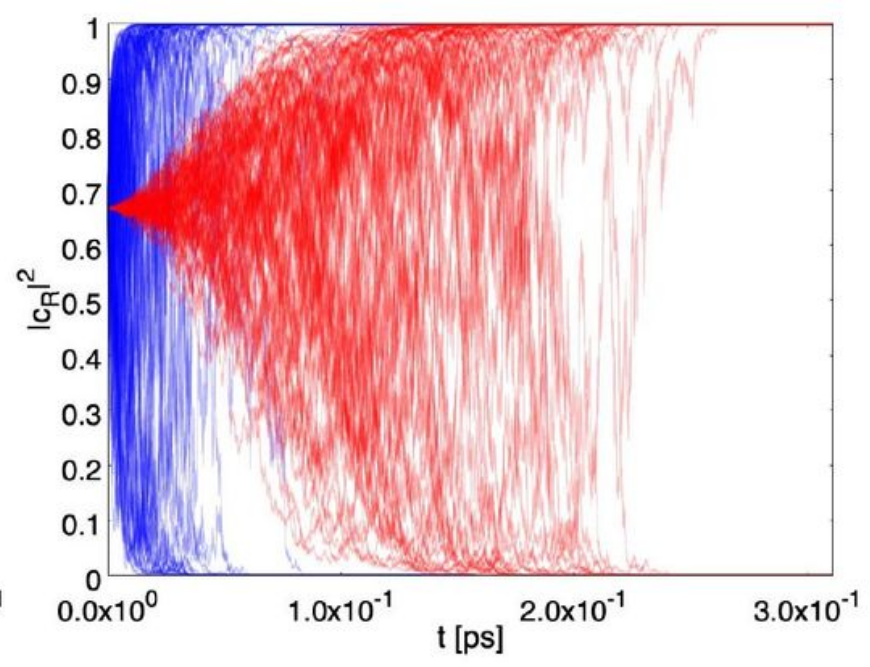

(b) Starting condition: $\left|c_{R}\right|^{2}=\frac{2}{3}$.

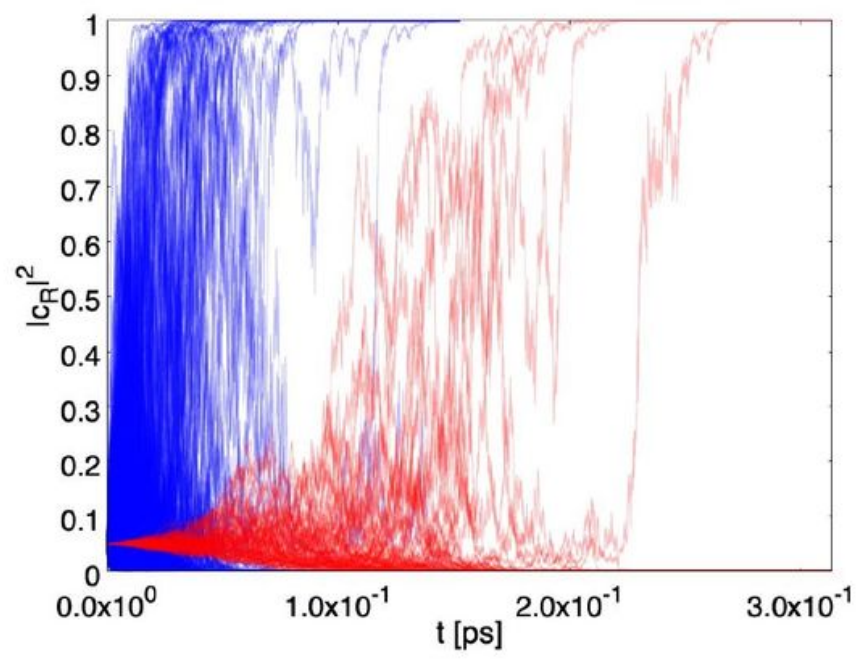

(d) Starting condition: $\left|c_{R}\right|^{2}=\frac{1}{20}$.

\section{Figure 5}

Please see the Manuscript PDF file for the complete figure caption 


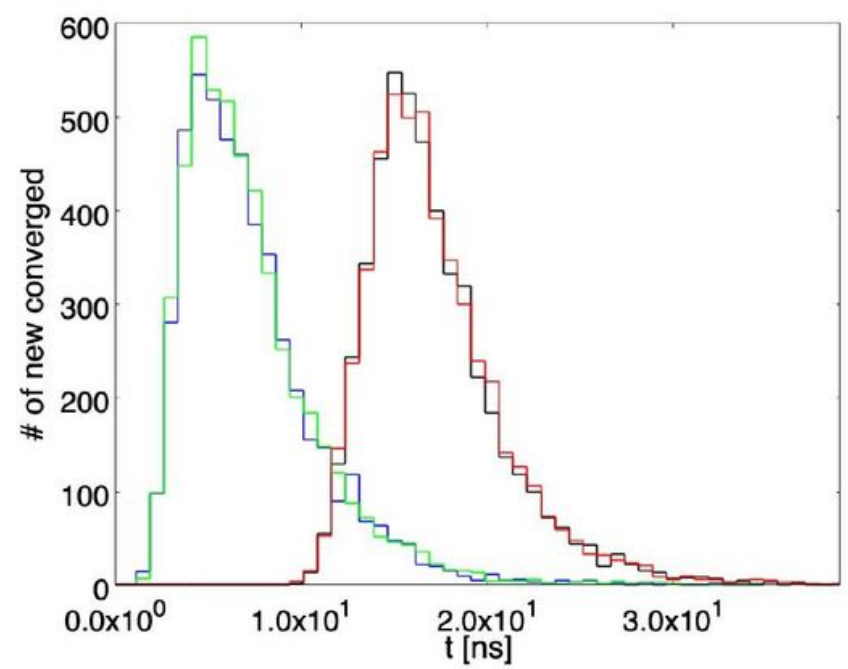

(a) Starting condition: $\left|c_{R}\right|^{2}=\frac{1}{2}$.

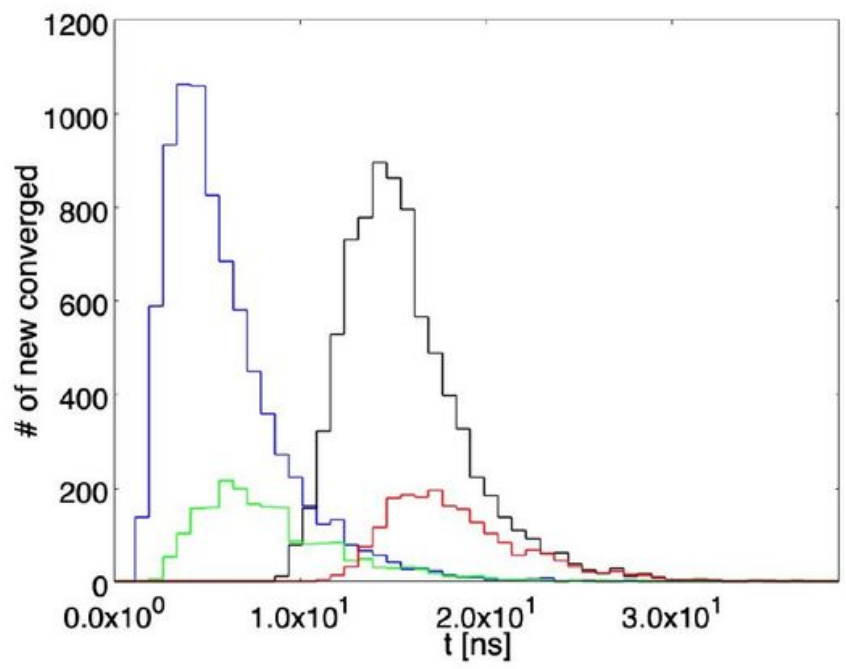

(c) Starting condition: $\left|c_{R}\right|^{2}=\frac{4}{5}$.

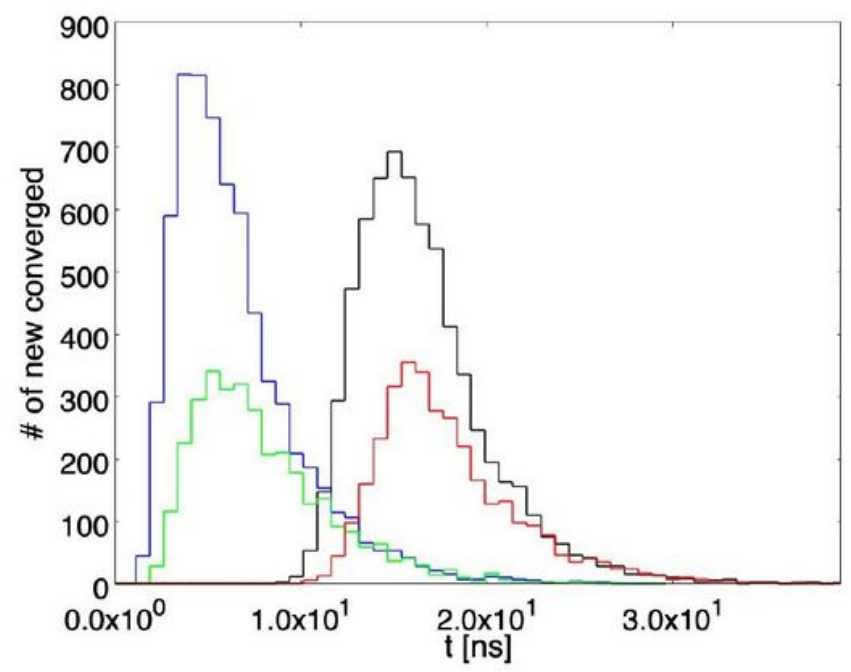

(b) Starting condition: $\left|c_{R}\right|^{2}=\frac{2}{3}$.

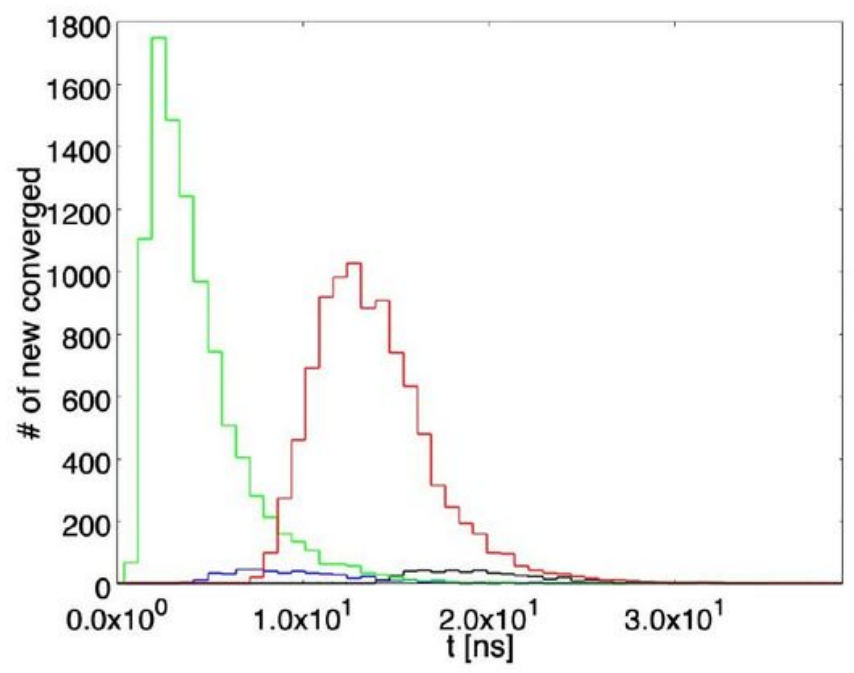

(d) Starting condition: $\left|c_{R}\right|^{2}=\frac{1}{20}$.

Figure 6

Please see the Manuscript PDF file for the complete figure caption 


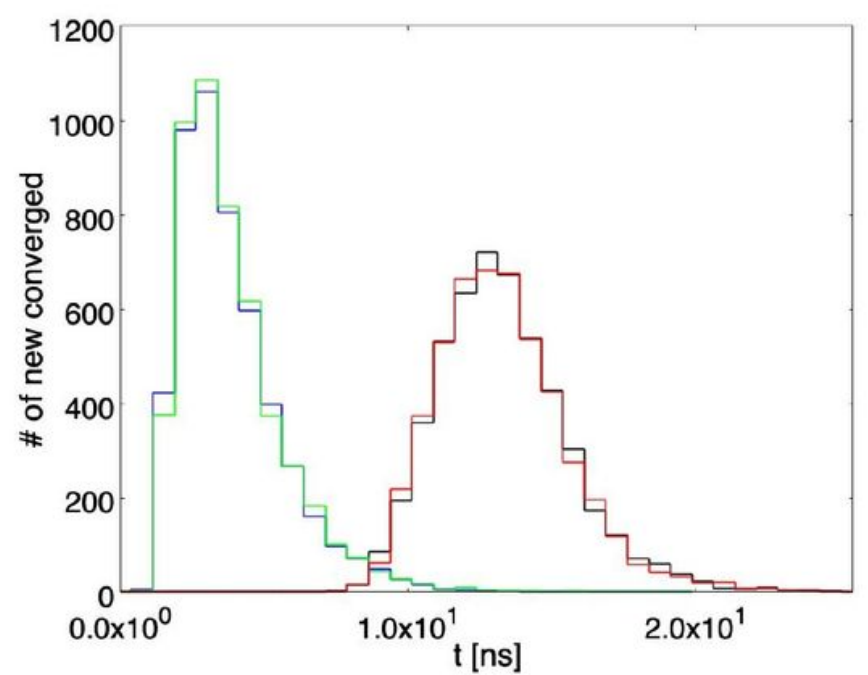

(a) Starting condition: $\left|c_{R}\right|^{2}=\frac{1}{2}$.

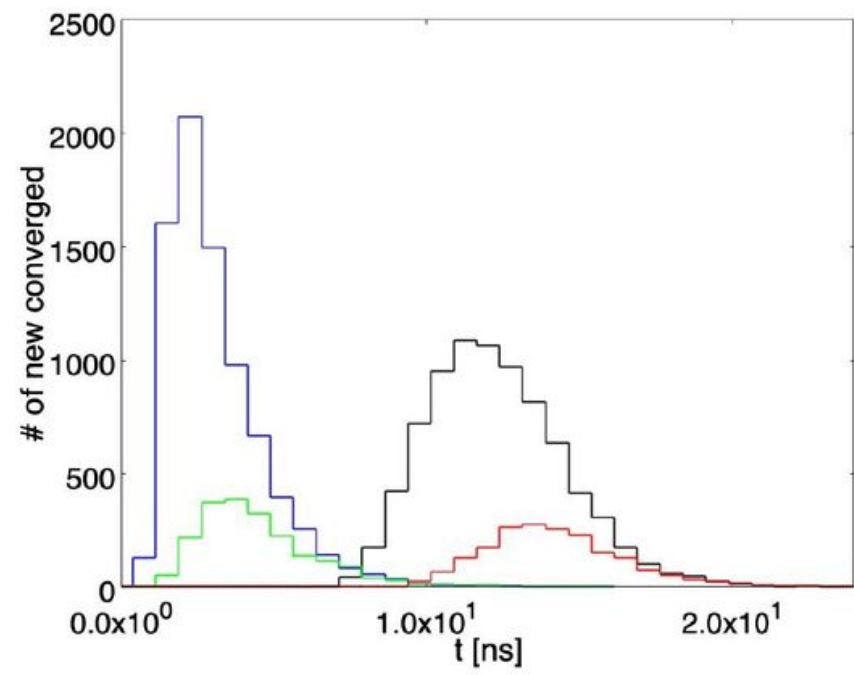

(c) Starting condition: $\left|c_{R}\right|^{2}=\frac{4}{5}$.

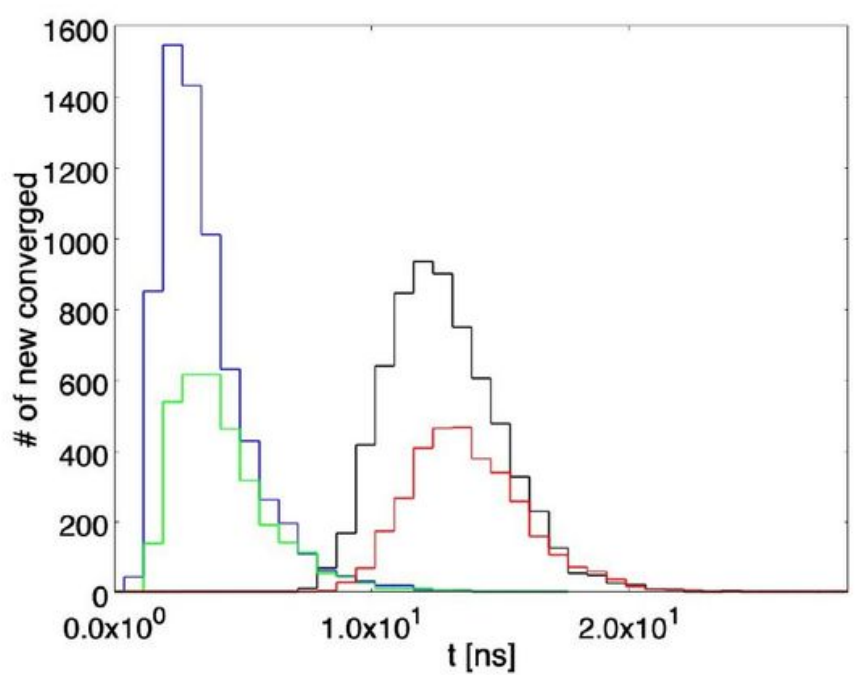

(b) Starting condition: $\left|c_{R}\right|^{2}=\frac{2}{3}$.

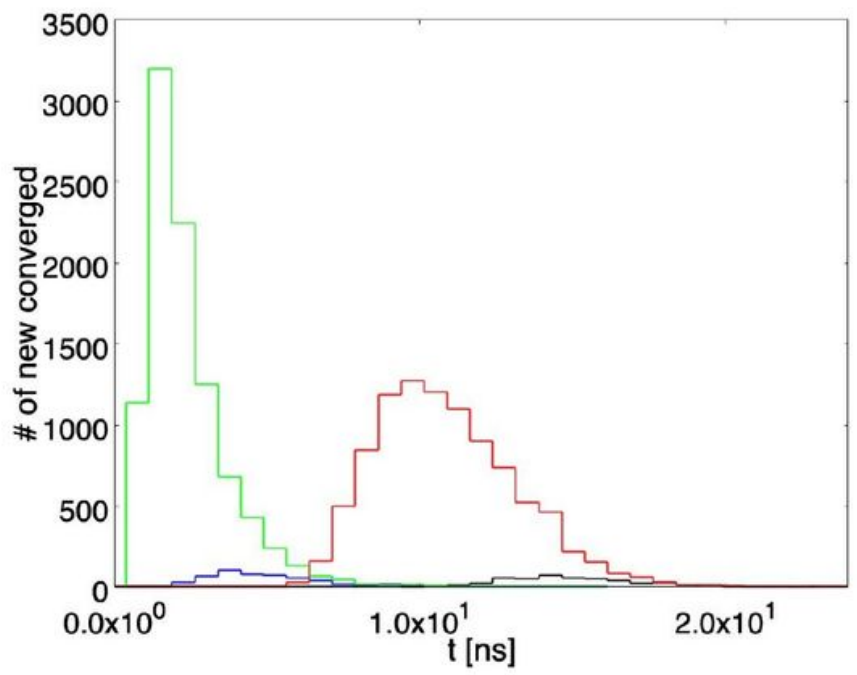

(d) Starting condition: $\left|c_{R}\right|^{2}=\frac{1}{20}$.

\section{Figure 7}

Please see the Manuscript PDF file for the complete figure caption 


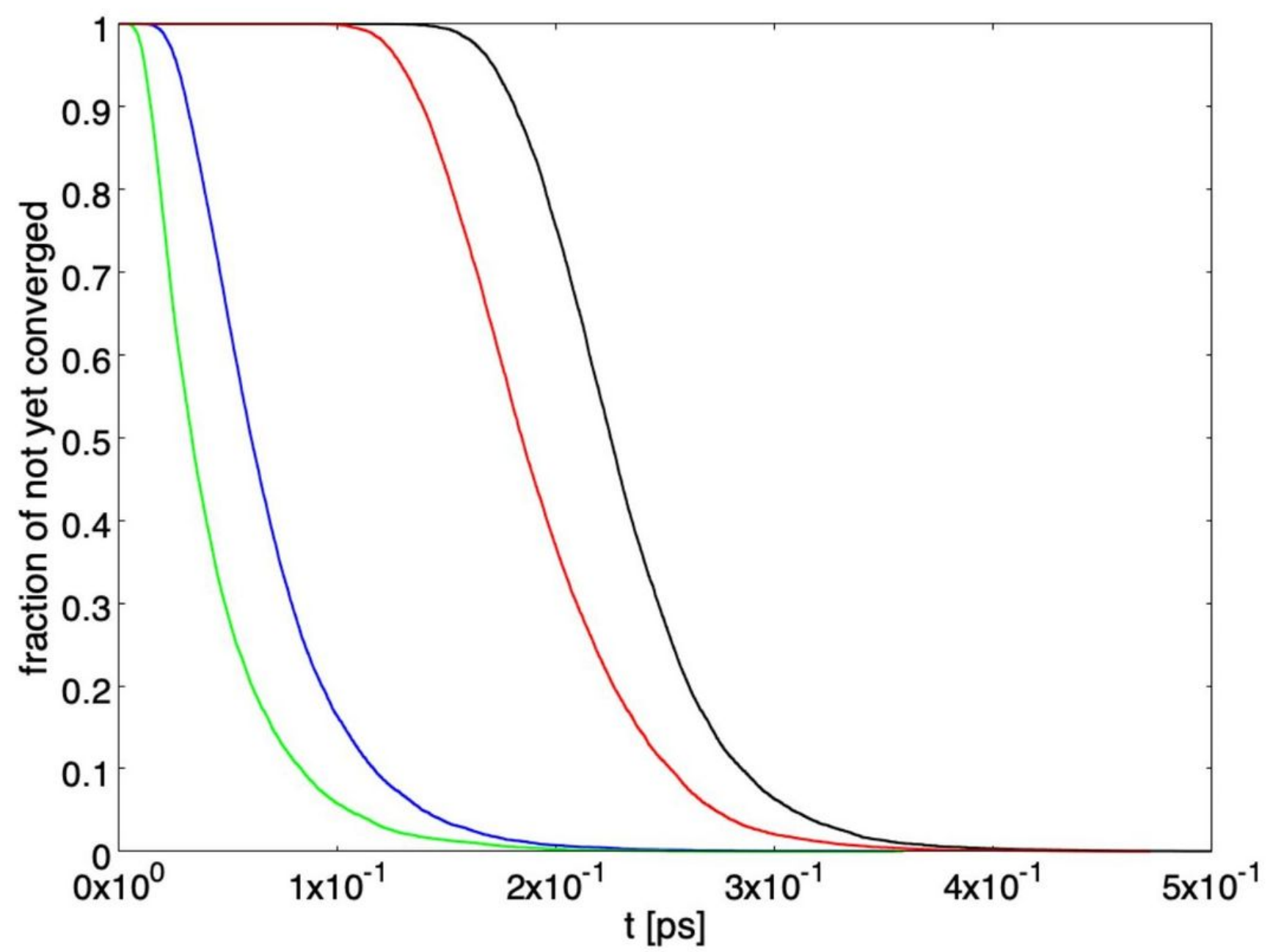

Figure 8

Please see the Manuscript PDF file for the complete figure caption 


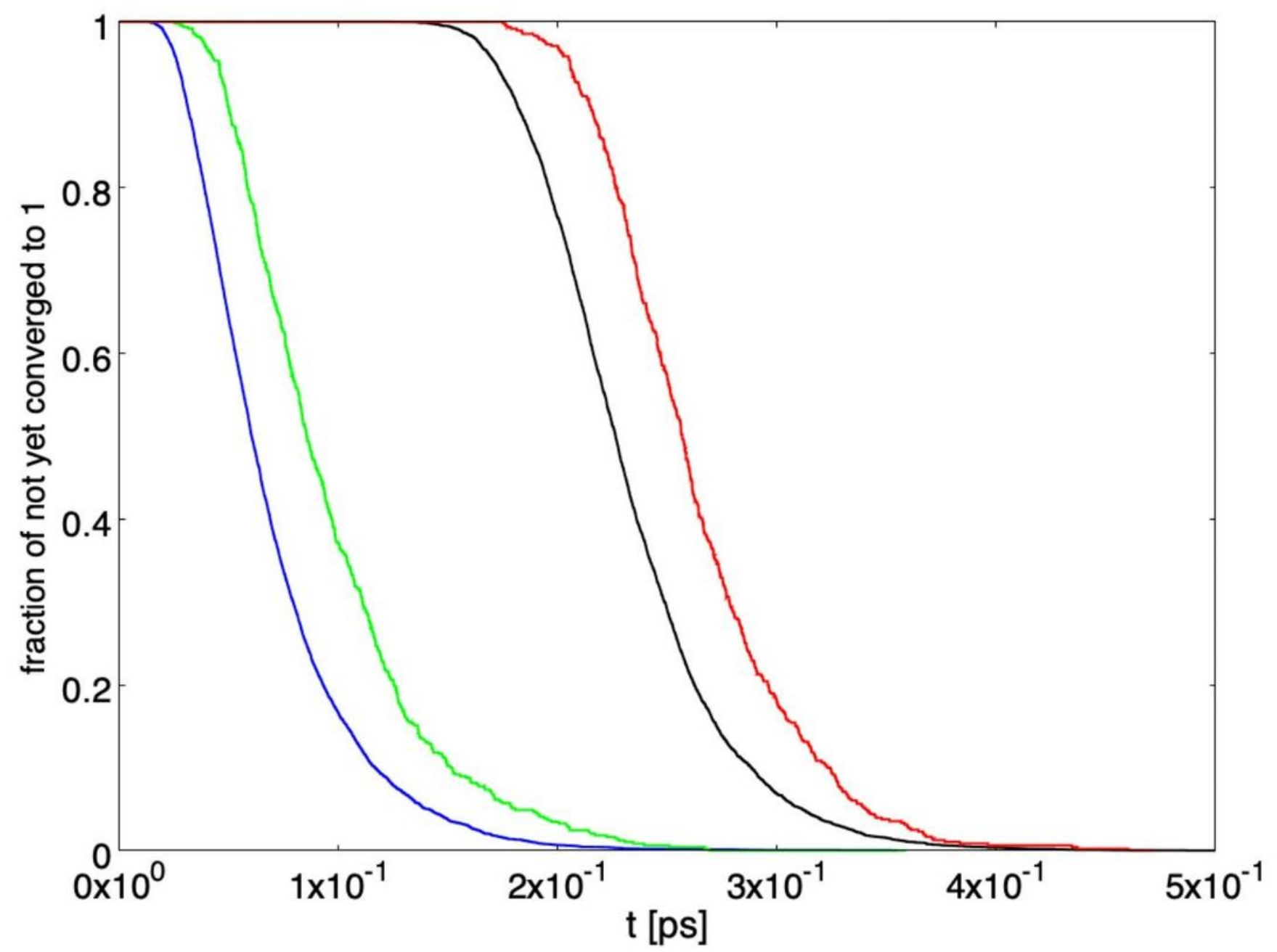

Figure 9

Please see the Manuscript PDF file for the complete figure caption 